\title{
Pairing and Density Correlations of Stripe Electrons in a Two-Dimensional Antiferromagnet
}

\author{
Henrik Johannesson \\ Institute of Theoretical Physics, \\ Chalmers University of Technology and Göteborg University, \\ SE-412 96 Göteborg, Sweden \\ G. I. Japaridze \\ Andronikashvili Institute of Physics, \\ Georgian Academy of Sciences, \\ Tamarashvili str. 6, Tbilisi 38007\%, Georgia
}

(Dated: January 30, 2003)

\begin{abstract}
We study a one-dimensional electron liquid embedded in a 2D antiferromagnetic insulator, and coupled to it via a weak antiferromagnetic spin exchange interaction. We argue that this model may qualitatively capture the physics of a single charge stripe in the cuprates on length- and time scales shorter than those set by its fluctuation dynamics. Using a local mean-field approach we identify the low-energy effective theory that describes the electronic spin sector of the stripe as that of a sine-Gordon model. We determine its phases via a perturbative renormalization group analysis. For realistic values of the model parameters we obtain a phase characterized by enhanced spin density and composite charge density wave correlations, coexisting with subleading triplet and composite singlet pairing correlations. This result is shown to be independent of the spatial orientation of the stripe on the square lattice. Slow transverse fluctuations of the stripes tend to suppress the density correlations, thus promoting the pairing instabilities. The largest amplitudes for the composite instabilities appear when the stripe forms an antiphase domain wall in the antiferromagnet. For twisted spin alignments the amplitudes decrease and leave room for a new type of composite pairing correlation, breaking parity but preserving time reversal symmetry.
\end{abstract}

PACS numbers: 71.27.+a, 71.10.Hf, 74.20.Mn

\section{INTRODUCTION}

Extensive experimental studies - including elastic and inelastic neutron scattering ${ }^{1}, \mathrm{ARPES}^{2}, \mu \mathrm{SR}^{3}$, and NMR experiments $\underline{\underline{4}}$ - have confirmed that stripe formation is a property common to most high- $\mathrm{T}_{c}$ cuprates. In the underdoped regime, at some critical hole doping, the mobile holes segregate into an array of "stripes" that slice the copper-oxide planes into alternating phase - antiphase antiferromagnetic domains. The stripes coexist with superconductivity, but as one enters the overdoped region they begin to evaporate, signaling a crossover to a conventional metal with a uniform charge distribution. Significantly, stripe phases are observed also in other doped antiferromagnets, such as the "Nickelates" $\underline{5}$ and the "Manganites" $\underline{\underline{6}}$ (colossal magnetoresistance materials, where the "stripes" are actually 2D sheets of holerich regions). This suggests that stripe formation is a robust and generic property of this class of matter. Still, the basic questions why stripes form and what role they play for superconductivity in the cuprates remain controversial.

Early mean-field calculations on the 2D Hubbard mode ${ }^{17}$ suggested that the stripe phase is due to the reduction in kinetic energy of holes propagating transverse to the stripes. In this approach, however, the possible connection to superconductivity is left unanswered. In an alternative approach ${ }^{8}$, it is argued that stripes form as a response to the competition between long-range Coulomb interactions (which push the holes apart) and short-range antiferromagnetic interactions (which tend to "phase separate" the holes into a single region). Within this scenario it has been argued that a proposed spin gap from the undoped domains is transmitted to the stripes via pair hopping ("spin gap proximity effect" ing to enhanced charge density wave (CDW) as well as superconducting pairing correlations along the stripes. For static stripes (as seen in e.g. in the Nickelates, or the Nd-doped $\mathrm{La}_{2-x} \mathrm{Sr}_{x} \mathrm{NdCuO}_{4}$ ) the CDW correlations dominate. In the presence of transverse stripe fluctuations, however, these appear to die out ${ }^{10}$, possibly opening a door to superconductivity. Other scenarios, where the stripes actually compete with superconductivity, have also been proposed 11 .

Most of the theoretical attempts to explore the properties of stripes model these as a collection of onedimensional (1D) or quasi-1D electron liquids 12 , coupled to their neighbors ${ }^{13}$, or to an insulating background, either via pair hopping of charge carriers (as in the "spin gap proximity effect" $\underline{-1}$ ) or by a spin exchange. The various spin-exchange scenarios that have been suggested ${ }^{14.15 .16}$ also predict that a spin gap opens in the spectrum of the stripe electrons, signaling enhanced superconducting fluctuations along the stripes. In fact, it is common to find a dynamically generated spin gap for a one-dimensional electron gas (1DEG) coupled to an active environment ${ }^{9,17.18 .19,20}$, of which an antifer- 
romagnet is a particular realization $14,15,16,21,22,23,24$.

The simplest such model is maybe that of the $1 \mathrm{D}$ Kondo-Heisenberg lattice (KHL) which consists of a 1DEG interacting weakly with an antiferromagnetic Heisenberg spin-1/2 chain by a Kondo coupling. Away from half-filling this model has a spin gap 22.23 and one thus expects the presence of superconducting correlations. Indeed, it was shown recently 25,26 that the spin gap supports composite ${ }^{27,28}$ odd-frequency odd-parity singlet pairing ${ }^{29}$ as well as a composite CDW. A generalization of this model that may mimic stripe physics more closely is that of a 1DEG coupled by a Kondo coupling to two non-interacting antiferromagnetic Heisenberg spin$1 / 2$ chains, together emulating the insulating background in which a stripe is embedded. Rather surprisingly, as shown recently ${ }^{30}$, this generalized model has no spin gap but instead renormalizes to a fixed point belonging to the class of chirally stabilized electron liquids ${ }^{31}$. Still, the model exhibits the same unconventional pairing instabilities as found for the 1D KHL ${ }^{25.26}$.

A "strong" interpretation of the results in Refs. 25,26 and 30 may seem to exclude spin exchange as a possible source of the spin gap in the high- $\mathrm{T}_{c}$ cuprates: oddfrequency pairing appears difficult to reconcile with the experimental observation that superconductivity in these compounds is due to d-wave BCS paired electrons. However, the recent report ${ }^{32}$ that underdoped BSCCO breaks time reversal symmetry - in the "normal" as well as the superconducting state - cautions us that the case may not be closed. The time reversal breaking is seen below a temperature $T_{\text {gap }}$ at which a pseudogap 33 opens, suggesting that it is connected with some order parameter that develops enhanced correlations below this characteristic temperature ${ }^{34}$. It has been argued that the pseudogap in the cuprates may be identified with the amplitude of the pairing order parameter, with long-range superconducting order appearing at the onset of global phase coherence (carried by Josephson tunneling of pairs between the stripes $)^{35}$. One may envision a variant of this scenario where spin exchange between the stripes and their environment (maybe in conjunction with pair hopping) supports two coexisting types of quasi-one dimensional pairing correlations below $T_{\text {gap }}$, one of which breaks time reversal. As one approaches the superconducting transition, the enhanced stripe fluctuations may favor the other type (which could re-emerge as long-range d-wave order via the dimensional crossover ${ }^{35}$ implied at $T_{c}$ ), while the channel that exhibits time-reversal breaking remains incoherent, with only finite-range correlations present. Although speculative only, the viability of this brand of scenario can be judged only by more closely examining the physics driven by a stripe-environment spin-exchange interaction. This is the purpose of our paper.

We shall consider an extended version of the model in Ref. 16, where a 1D electron liquid (representing a single stripe) is embedded in a $2 \mathrm{D}$ antiferromagnetic background, and coupled to it via an antiferromagnetic spin exchange. We show that this setup leads to a spin-gap phase for the electrons on the stripe, and we identify its leading instabilities. We further address the question to what extent the instabilities found are sensitive to the relative orientation of the staggered magnetizations on each side of the stripe. In the simplest case of a sitecentered stripe ${ }^{37}$ the spin alignment across the stripe is antiferromagnetic (phase-antiphase domains). However, the alignment is not expected to be perfect and it is therefore important to check the stability of the spin gap phase w.r.t. deviations from the phase-antiphase orientation of the magnetic domains separated by the stripe. In addition, we shall explore the issue whether the spatial orientation of the stripe on the underlying lattice may influence the stripe electron dynamics when the dominant interaction with the environment is that of a spin exchange.

This latter question is of particular relevance considering recent experimental findings of "diagonal stripes" in the underdoped glassy phase of the cuprates. As discovered by Wakimoto et $a l^{38}$, the insulating $\mathrm{La}_{1.95} \mathrm{Sr}_{0.05} \mathrm{CuO}_{4}$ exhibits sharp two-dimensional elastic magnetic peaks at $(\pi \pm \epsilon, \pi \pm \epsilon$ ) (in tetragonal square lattice notation, with $\epsilon \sim x \approx 0.05, x$ being the doping level). Assuming that the magnetic peaks are associated with charge stripe order ${ }^{36}$, this implies that static stripes run along the diagonal of the square $\mathrm{Cu}^{2+}$ lattices that make up the $\mathrm{CuO}$-planes in this compound. This is in exact analogy to the diagonal static stripe structure seen (and theoretically predicted ${ }^{7}$ ) in the insulating Nickelate $\mathrm{La}_{2-x} \mathrm{Sr}_{x} \mathrm{NiO}_{4+x}$, but different from the structure in superconducting $\mathrm{La}_{2-x} \mathrm{Sr}_{0.05} \mathrm{CuO}_{4}$ (with $x>0.05$ ) where the stripes are oriented along the copper-oxide bonds ("collinear stripes"). Very recently, these finding were extended to the full insulating spin glass phase in $\mathrm{La}_{2-x} \mathrm{Sr}_{x} \mathrm{CuO}_{4}(0.02 \leq x \leq 0.055)^{39}$. Thus, the onset of superconductivity in the low-temperature underdoped region appears to proceed via a stripe rotation by $45^{\circ}$, from a diagonal to a collinear stripe configuration. In the case of a collinear (site-centered) structure, a stripe is embedded in a local antiferromagnetic background. In contrast, in the diagonal structure the stripe electrons experience a local ferromagnetic environment. In both cases the antiferromagnetic domains are $\pi$-shifted across the stripes, and it is a priori not clear whether the different local orderings along the stripes influence the electron dynamics differently. To clarify the situation requires a careful study, and we here make a first attempt on it.

To isolate the core of the problem we shall make a few simplifying assumptions:

(i) We study the electron dynamics on a single stripe, and, in the first part of our analysis, neglect its possible coupling to neighboring stripes. Moreover, the stripe is taken to be static. This implies that for a fluctuating stripe (as typically seen in a superconducting phase) we can only hope to capture processes on length- and time scales shorter than those set by its fluctuation dynamics. 
This is expected to be much slower than the dynamics of charge carriers along the stripe: The latter appear on an energy scale $\sim 1 \mathrm{eV}$, whereas the stripe fluctuations are coordinated with those of the localized spins, at a scale $\sim 1-10 \mathrm{meV}$. Having obtained the characteristic features of a single static stripe we then add "by hand" the transverse fluctuations and interstripe couplings, and study their effect on the pairing- and density correlations of the stripe electrons.

(ii) We model the stripe - at Fermi momenta incommensurate with the underlying lattice - as a one-dimensional metallic wire. Thus, we assume that the disorder (from e.g. dopant potentials) is sufficiently weak so that localization effects set in on length scales much larger than those that we probe here.

(iii) There is an important distinction between sitecentered and bond-centered stripes ${ }^{37}$ : The spin alignment across an anti-phase domain wall is antiferromagnetic for a site-centered stripe and ferromagnetic for a bond-centered stripe (which has a finite width). Motivated by recent theoretical work ${ }^{40}$ where bond-centered configurations appear to be inconsistent with ARPES studies of $\mathrm{La}_{2-x} \mathrm{Sr}_{x} \mathrm{CuO}_{4}$, , we here focus on the simplest case of a site-centered charge stripe, to be described by a 1D Hubbard model. We shall explore elsewhere the case of bond-centered stripes, building on the corresponding analysis by Krotov, Lee and Balatsky $\stackrel{15}{\underline{15}}$ of a Hubbard ladder in an antiferromagnetic environment.

(iv) As suggested by neutron scattering data on the relevant materials 1 , the environment is Néel ordered up to some characteristic scale (which, in the relevant temperature range, is much larger than the linear dimension of a stripe), with a $\pi$-shift across the stripe when this is site centered (phase - antiphase domains). In our formal analysis we depict each Néel ordered domain as a semi-infinite 2D Heisenberg antiferromagnet, and ignore possible topological effects that may be present for finitewidth insulating domains, or "spin ladders" 42 . We shall give precise estimates for the range of validity of this approximation, thus establishing its physical relevance.

(v) As we have already discussed, we couple the stripe electrons to its insulating environment exclusively through a spin exchange interaction. Given that the Fermi momentum of the stripe is incommensurate with that of any low-lying excitation of the environment, excursions of single charge carriers is a process that violates momentum conservation, and hence is suppressed (on the time scales defined in (i)). Pair hopping is still allowed, provided that the pair carries zero total momentum. As suggested by the analysis in Ref. 9, pair hopping is favored as a dominant process when the low-lying spin excitations of the environment are gapped ${ }^{43}$. When such a gap is absent, as is the case when the environment is Néel ordered, the virtual hybridization between delocalized levels on the stripe and the localized levels in the environment produces an effective spin exchange that is expected to compete effectively with pair hopping. Here, we focus on the effect of the spin exchange. (vi) We limit our attention to the physically relevant case of a weak spin exchange $J_{K}$ between stripe and environment, $0<J_{K} \ll J_{H}$, where $J_{H}$ is the antiferromagnetic exchange between the localized spins in the environment. This allows us to treat the problem in a continuum limit 23 .

(vii) Finally, we stress that finite-size or boundary effects of the 1D electrons 44 are not included in our analysis. As the stripes in the cuprates are mesoscopic structures ${ }^{45}$, these effects should in principle be taken into account. However, as they are not expected to qualitatively change the conclusions arrived at in the largedistance limit considered here, we leave this study for the future.

Clearly, by assumptions (i) - (vii) we loose several facets of the full problem. Still, we believe that our "stripped-down" approach has its merits: Not only does it isolate and expose a crucial element of "stripe physics", but as we shall show, it allows us to perform a wellcontrolled analytical study, producing results that can be taken as a reliable starting point for more realistic studies. Moreover, the problem as defined by (i)-(vii) is important in its own right, and is of relevance to the more general issue of one-dimensional electron liquids in active environments9.17.18.19.20. This is a central problem in the theory of correlated electrons, motivated by experiments on quasi-1D organic conductors $\$ 46$, nanowires 47 , and edge states in quantum Hall systems $\frac{48}{}$.

Given the assumptions (i) - (vii), we model the stripe by an extended $(U-V)$ Hubbard chain, weakly coupled to a phase - antiphase antiferromagnetic environment by a Kondo lattice interaction. Treating the Hubbard chain via standard bosonization while describing the environment by a nonlinear $\sigma$-model, we follow the approach introduced in Ref. 16 and exploit the symmetry breaking in the magnetic environment to "absorb" the Kondo lattice interaction as an effective spin-spin interaction among the stripe electrons. In this way we obtain an effective low-energy model for the stripe electrons decoupled from the environment - and accessible to a well-controlled perturbative renormalization group analysis. This allows us to pinpoint the dynamic instabilities in the low-energy, weak-coupling $\left(J_{K} \ll J_{H}<|U|,|V|\right)$ limit.

Our most important results can be summarized:

- For realistic values of the model parameters, and with a phase-antiphase Néel-configuration across the stripe, an electronic spin gap opens on the stripe with a spin-density and a composite chargedensity wave as the leading instabilities. The subleading instability is that of conventional triplet pairing, coexisting with composite singlet pairing (which breaks parity and time reversal). Slow transverse stripe fluctuations tend to suppress the density correlations, thus promoting the pairing instabilities.

- The low-energy physics is insensitive to the spatial 
orientation of the stripe on the lattice: The results summarized above hold for both collinear and diagonal stripes (with the possible exception that the composite singlet pairing is suppressed for a diagonal stripe).

- The instabilities found for the phase-antiphase Néel-configuration are still present when the relative orientation of the staggered magnetizations on the respective sides of a (collinear) stripe has been twisted by an arbitrary angle. In addition, the twist allows for a novel type of composite pairing correlations to appear, respecting time reversal but breaking parity.

The paper is organized as follows: In Sec. II we introduce the lattice models for site-centered collinear and diagonal stripes coupled to a phase-antiphase environment by a Kondo interaction, and derive the corresponding low-energy effective actions. In Sec. III we perform an RG analysis and identify the order parameter correlations along the stripes that get enhanced by the spin exchange. In Sec IV we then study - for the case of a collinear stripe - the stability of these correlations w.r.t. perturbations of the relative orientation of the spin alignments across the stripe. Sec. IV, finally, contains a summary and a brief discussion of our results. Throughout the paper we try to supply sufficient information to make the analysis accessible also to the non-expert.

\section{THE MODEL}

For clarity, we shall treat the collinear and diagonal stripe configurations separately. The effective low-energy theories that emerge in the two cases are essentially the same (with certain provisos), but to arrive at this result requires some care. Much of the analysis builds upon well-known results, but in order to make the exposition self-contained we outline the most important points. Also, some key elements are new or need particular attention.

\section{A. Collinear Stripes}

We represent the stripe (running, say, along the $\mathrm{x}-$ direction of a square lattice) by an extended $(U-V)$ Hubbard chain, $H_{\text {Hubbard }}$, coupled via a Kondo lattice interaction $H_{K o n d o}$ to the nearest localized spins on each side of the stripe. These spins, like the rest of the localized spins, interact mutually via an antiferromagnetic nearest-neighbor Heisenberg spin exchange $H_{A F M}$, and reside in one of the two semi-infinite antiferromagnetic domains that surround the stripe, denoted $A$ and $B$ respectively (see FIG. 1).

The $A$ and $B$ domains are assumed to be antiferromagnetically ordered, and correlated via a $\pi$-shift across the stripe (phase - antiphase domains) but there is no direct

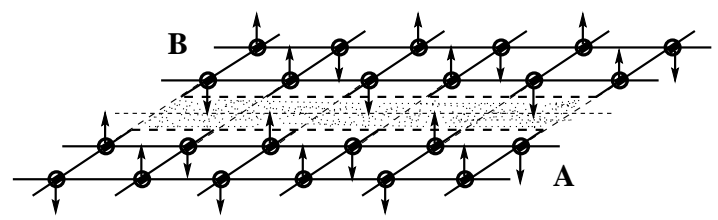

FIG. 1: Collinear stripe structure.

interaction between $A$ and $B$ spins ${ }^{49}$. Thus, we study the lattice model

$$
H=H_{\text {Hubbard }}+\sum_{i=A, B}\left(H_{A F M}^{(i)}+H_{\text {Kondo }}^{(i)}\right)
$$

where

$$
\begin{gathered}
H_{\text {Hubbard }}=-t \sum_{r, \alpha}\left(c_{r+1, \alpha}^{\dagger} c_{r, \alpha}+h . c .\right) \\
+U \sum_{r} \hat{n}_{r, \uparrow} \hat{n}_{r, \downarrow}+V \sum_{r} \hat{n}_{r} \hat{n}_{r+1}, \quad(2) \\
H_{A F M}^{(i)}=J_{H} \sum_{r, j^{(i)}}\left(\boldsymbol{S}_{r, j^{(i)}} \cdot \boldsymbol{S}_{r+1, j^{(i)}}+\boldsymbol{S}_{r, j^{(i)}} \cdot \boldsymbol{S}_{r, j^{(i)}+1}\right), \\
J_{H}>0, \quad i=A, B, \quad(3) \\
H_{\text {Kondo }}=J_{K} \sum_{r, \alpha, \beta} c_{r, \alpha}^{\dagger} \boldsymbol{\sigma}_{\alpha \beta} c_{r, \beta} \cdot\left(\boldsymbol{S}_{r, j^{(A)}=1}+\boldsymbol{S}_{r, j^{(B)}=1}\right), \\
J_{K}>0 . \quad(4)
\end{gathered}
$$

Here $c_{r, \alpha}$ is a stripe electron operator at site $r$ with spin index $\alpha=\uparrow, \downarrow, \hat{n}_{r, \alpha}=c_{r, \alpha}^{\dagger} c_{r, \alpha}$ is the number operator, $\hat{n}_{r}=\sum_{\alpha} \hat{n}_{r, \alpha}$ is the density operator, and $\boldsymbol{S}_{r, j(i)}$ is the operator for a localized spin at a lattice site with coordinate $r\left(j^{(i)} \geq 1\right)$ in a direction parallel with (transverse to) the stripe. The vector of Pauli matrices is denoted by $\boldsymbol{\sigma}$, and we have absorbed a factor of $1 / 2$ into the coupling constant $J_{K}$. Note that we have included a nearestneighbor interaction in $H_{\text {Hubbard }}$ to qualitatively account for the poor screening of the Coulomb interaction from the insulating environment. The Coulomb-driven on-site and nearest-neighbor coupling constants are typically repulsive $U, V>0$. However, in what follows we will treat these parameters as effective (phenomenological) ones, and assume that they include all possible contributions and renormalizations coming from the interaction between stripe electrons and the nonmagnetic degrees of freedom of the environment, such as the electron-phonon coupling, or coupling to other electronic subsystems in the environment ${ }^{50}$. As implicit in Eq. (4), we use the convention that the transverse coordinates take values $j^{(A)}=j^{(B)}=1$ on the $A$ - and $B$-arrays adjacent to the stripe. When convenient, we use the compact notation $\boldsymbol{S}_{r}^{(i)} \equiv \boldsymbol{S}_{r, j^{(i)}}(i=A, B)$ for the spins on these arrays. By assumption (vi) in Sec. I, the antiferromagnetic Kondo 
lattice coupling $J_{K}$ is weak, i.e. $J_{K} \ll J_{H}$, $t$, allowing us to keep only the low-energy sectors of the stripe and the antiferromagnetic domains when analyzing its effect.

The model in Eq. (1) is a modified version of that in Ref. 16 by having a coupling of two semi-infinite $2 \mathrm{D}$ antiferromagnetic domains to the stripe, one on each side of it, in this respect mimicking the geometry "seen" by a real stripe. As a consequence, with the assumption that the Néel order of the $A$ and $B$ domains are $\pi$-phase shifted relative to each other, we will be able to treat the metallic as well as the Mott insulating case (half-filled Hubbard band) within the same formalism. This is different from the model in Ref. 16, where the assumption of a metallic stripe was crucial. Note that by turning off $U$ and $V$ in (2) and keeping only one array of localized spins, say, in the $A$-domain, the Hamiltonian in (11) collapses to the one-dimensional Heisenberg-Kondolattice model (HKL) ${ }^{51}$. This model has recently attracted a great deal of attention ${ }^{22.23}$ and we shall connect back to it when discussing our results in Sec. IV. We should here emphasize that having "built in" the presence of stripes into the model, we cannot address the issue of what actually triggers the stripe formation. For this, one must turn to other approaches, some of them mentioned above $e^{7.8 .11}$. A recent attack on the problem of stripe formation has made use of a spin-fermion model $\stackrel{52}{ }$ which has the same Hamiltonian structure as (1), but with the difference that there is no constraint on the mobility of the electrons in (2). In other words, the doped holes are now free to hop around on the 2D lattice. Monte Carlo simulations on the model suggest that the holes self-organize into onedimensional charge stripes separating insulating spin domains (phase shifted by $\pi$ across the stripes). This is the starting point when writing down our model in (11).

Given the Hamiltonian in (1), its partition function can be written as a path integral,

$$
Z=\int \mathcal{D}[c] \mathcal{D}\left[c^{\dagger}\right] \mathcal{D}\left[\boldsymbol{\Omega}_{A}\right] \mathcal{D}\left[\boldsymbol{\Omega}_{B}\right] e^{-\mathcal{S}\left[c^{\dagger}, c, \boldsymbol{\Omega}_{A}, \boldsymbol{\Omega}_{B}\right]}
$$

with a Euclidean action $\mathcal{S}$. The electron operators are here simulated by Grassmann numbers $\left(c_{r, \alpha}^{\dagger}, c_{r, \alpha}\right)$, while the role of the localized spin operators $\boldsymbol{S}_{r, j^{(i)}}$ are played by vectors $S \boldsymbol{\Omega}_{r, j^{(i)}}(i=A, B)$ which parameterize states in a coherent spin representation ${ }^{53}$. Note that we have here used the short-hand notation $c \equiv\left\{c_{r, \alpha}\right\}$ and $\boldsymbol{\Omega}_{i} \equiv$ $\left\{\boldsymbol{\Omega}_{r, j^{(i)}}\right\}, i=A, B$. In the limit of large spin, $S \rightarrow \infty$, only diagonal matrix elements of a spin Hamiltonian survive in this representation. This makes the large- $S$ coherent state representation an efficient tool to mold a quantum partition function into a path integral. To retain quantum effects, however, present for physical values of the spin $(S=1 / 2$ in the case of the cuprates), it is crucial to keep also non-diagonal matrix elements in the construction of $S$. These produce a sum over Berry phases and contain a memory of the intrinsic quantum nature of the spins. The procedure is standard ${ }^{54}$, and one obtains the action

$$
\begin{aligned}
\mathcal{S} & =\int_{0}^{\beta} d \tau\left(\sum_{r} c_{r, \alpha}^{\dagger} \partial_{\tau} c_{r, \alpha}+H\left(c^{\dagger}, c, S \boldsymbol{\Omega}_{A}, S \boldsymbol{\Omega}_{B}\right)\right) \\
& +i S \sum_{i=A, B} \sum_{r, j^{(i)}} \Phi_{r, j^{(i)}}
\end{aligned}
$$

where $\tau$ corresponds to inverse temperature so that $0<$ $\tau<\beta$ and the spin (Grassmann) fields are periodic (antiperiodic) in $\tau$. For the purpose of studying the lowenergy dynamics we confine our attention to the zerotemperature limit $(\beta \rightarrow \infty)$. The third term in (6) is precisely the sum over Berry phases

$$
\Phi \boldsymbol{r}_{i}=\oint_{\Gamma \boldsymbol{r}_{i}} d \boldsymbol{\Omega} \boldsymbol{r}_{i} \cdot \mathbf{A}\left(\boldsymbol{\Omega} \boldsymbol{r}_{i}\right),
$$

one for each spin attached at site $\left(r, j^{(i)}\right) \equiv \boldsymbol{r}_{i}, i=A, B$. Here $\Gamma \boldsymbol{r}_{i}$ is the closed loop traced out by $\boldsymbol{\Omega}_{\boldsymbol{r}_{i}}$ in the interval $[0, \beta \rightarrow \infty]$, with $\mathbf{A}\left(\boldsymbol{\Omega}_{\boldsymbol{r}_{i}}\right)=\left(1-\cos \theta_{i}\right)\left(\sin \theta_{i}\right)^{-1} \varphi_{i}$ at each site $\boldsymbol{r}_{i}$ playing the role of a vector potential of a unit magnetic monopole located at the center of the sphere $\left|\boldsymbol{\Omega}_{\boldsymbol{r}_{i}}\right|=1$, parameterized by the spherical angles $\theta_{i}$ and $\phi_{i}$. The "instantaneous" Hamiltonian term $H\left(c^{\dagger}, c, S \boldsymbol{\Omega}_{A}, S \boldsymbol{\Omega}_{B}\right)$ in (6) acts at (imaginary) time slice $\tau$ and is obtained from (11) by replacing electron and spin operators by the corresponding Grassmann fields $\left(c_{r, \alpha}^{\dagger}, c_{r, \alpha}\right)$ and classical vectors $\left(S \boldsymbol{\Omega}_{\boldsymbol{r}_{A}}, S \boldsymbol{\Omega}_{\boldsymbol{r}_{B}}\right)$, respectively.

Next, to obtain the low-energy continuum version of the Hamiltonian in (1) we shall first review the standard constructions for a Hubbard chain and a 2D Heisenberg model, and then elaborate on the more intricate Kondolattice interaction which couples the two subsystems.

\section{1. $1 D$ Electron Chain: Low-Energy Theory}

The low-energy (field theory) approach to the 1D (extended) Hubbard model in (2) is based on the assumption of weak electron-electron interactions. Thus, assuming that $|U|,|V| \ll t$, we linearize the spectrum around the two Fermi points $\pm k_{F}\left(k_{F}=n_{e} \pi / 2 a_{0}\right.$, where $a_{0}$ is the lattice spacing and $n_{e}$ is the electron density), and decompose the original lattice operators into right-moving $\left(R_{\alpha}\right)$ and left-moving $\left(L_{\alpha}\right)$ chiral components:

$$
\begin{aligned}
c_{r, \alpha} & \rightarrow \mathrm{e}^{i k_{F} r a_{0}} R_{\alpha}(r)+\mathrm{e}^{-i k_{F} r a_{0}} L_{\alpha}(r) \\
& \rightarrow \sqrt{a_{0}}\left[\mathrm{e}^{i k_{F} x} R_{\alpha}(x)+\mathrm{e}^{-i k_{F} x} L_{\alpha}(x)\right],
\end{aligned}
$$

where in the second line we have taken the continuum limit $r a_{0} \rightarrow x$. Defining local charge and spin densities

$$
\begin{aligned}
& J_{R}=: R_{\alpha}^{\dagger} R_{\alpha}:, \quad J_{L}=: L_{\alpha}^{\dagger} L_{\alpha}: \\
& \boldsymbol{J}_{R}=: \frac{1}{2} R_{\alpha}^{\dagger} \boldsymbol{\sigma}_{\alpha \beta} R_{\beta}:, \quad \boldsymbol{J}_{L}=: \frac{1}{2} L_{\alpha}^{\dagger} \boldsymbol{\sigma}_{\alpha \beta} L_{\beta}:
\end{aligned}
$$

with repeated spin indices summed over, and with the normal ordering : ... : taken w.r.t. the ground state of 
the free system, it is now straightforward to write down the low-energy continuum version of the $U-V$ Hubbard chain in (2). The weak interaction preserves the important property of spin-charge separation, and one can write the theory on the form $H_{\text {Hubbard }}=H_{c}+H_{s}$, where ${ }^{55}$ :

$$
\begin{gathered}
H_{c}=\frac{\pi v_{c}}{2} \int \mathrm{d} x\left\{: J_{R} J_{R}:+: J_{L} J_{L}:-g_{0 c} J_{L} J_{R}-g_{0 u} \delta_{1 n_{e}}\left(R_{\uparrow}^{\dagger} R_{\downarrow}^{\dagger} L_{\uparrow} L_{\downarrow}+h . c .\right)\right\}, \\
H_{s}=2 \pi \tilde{v}_{s} \int \mathrm{d} x\left\{: J_{R}^{z} J_{R}^{z}:+: J_{L}^{z} J_{L}^{z}:-\tilde{g}_{0 s} J_{L}^{z} J_{R}^{z}-\tilde{g}_{0 \perp}\left(J_{L}^{x} J_{R}^{x}+J_{L}^{y} J_{R}^{y}\right)\right\} .
\end{gathered}
$$

The velocities of the charge $(c)$ and spin $(s)$ excitations, governed by $H_{c}$ and $H_{s}$ respectively, are given by

$$
v_{c}=v_{F}+\frac{a_{0}(U+6 V)}{2 \pi}, \quad \tilde{v}_{s}=v_{F}-\frac{a_{0}(U-2 V)}{2 \pi},
$$

with $v_{F}=2 a_{0} t \sin \left(\pi n_{e} / 2\right)$ the Fermi velocity. The small dimensionless coupling constants in (11) and (12) are given by

$$
\begin{aligned}
& g_{0 c}=-a_{0}(U+6 V) / \pi v_{F}, \\
& g_{0 u}=-a_{0}(U-2 V) / \pi v_{F}, \\
& \tilde{g}_{0 s}=\tilde{g}_{0 \perp}=a_{0}(U-2 V) / \pi v_{F} .
\end{aligned}
$$

The Kronecker delta multiplying the Umklapp term in (11) signifies that this term survives the phase fluctuations (originating from the chiral decomposition in (8)) only for a half-filled electron band $\left(n_{e}=1\right)^{55}$. The transverse component of the spin current coupling in (12),

$$
\tilde{g}_{0 \perp}\left(J_{L}^{x} J_{R}^{x}+J_{L}^{y} J_{R}^{y}\right)=-\frac{1}{2} \tilde{g}_{0 \perp}\left(R_{\uparrow}^{\dagger} L_{\uparrow} L_{\downarrow}^{\dagger} R_{\downarrow}+\text { h.c. }\right),
$$

describe backscattering of electrons. We have marked the spin-sector parameters in (12), (13) and (14) by a "tilde" as a reminder that these will be modified when coupling the stripe to the localized spins in the environment. How this comes about is discussed next.

\section{Phase - Antiphase Antiferromagnetic Domains: Low-Energy Theory}

In the presence of antiferromagnetic correlations in the insulating domains - as seen experimentally in the stripe materials 1 - the partition function in (5) at low energies is dominated by paths with

$$
\boldsymbol{\Omega}_{\boldsymbol{r}_{i}}=\gamma_{\boldsymbol{r}_{i}}(-1)^{\delta_{i B}} \sqrt{1-a_{0}^{2} \ell^{2}\left(\boldsymbol{r}_{i}\right)} \boldsymbol{n}\left(\boldsymbol{r}_{i}\right)+a_{0} \boldsymbol{\ell}\left(\boldsymbol{r}_{i}\right) .
$$

Here $\gamma \boldsymbol{r}_{i}= \pm 1$ is the parity of the sublattice to which the site $\boldsymbol{r}_{i}$ belongs, the unit vector $\boldsymbol{n}$ (suppressing the coordinate $\boldsymbol{r}_{i}$ for ease of notation) represents the local direction of the Néel order parameter field, and $a_{0} \boldsymbol{\ell}$ is a small orthogonal ferromagnetic fluctuation component, i.e. $\left|a_{0} \ell\right| \ll 1$, with $\boldsymbol{n} \cdot \boldsymbol{\ell}=0$. The phase factor $(-1)^{\delta_{i B}}$ in (16) appears because from now on we take the Néel fields in the $A$ and $B$ domains to be $\pi$-shifted relative to each other. (The choice of reference vector in the staggering factor can be made arbitrarily, with no effect on the physics.) We here note that for a site-centered stripe embedded in a spin- $1 / 2$ environment current estimates predict that this is a viable assumption for stripe electron densities $n_{e}<0.6$ in the limit where $J_{K} \sim J_{H}{ }^{56.57}$. However, for $J_{K} \ll J_{H}$, as assumed here, the critical density is expected to be larger.

With $\boldsymbol{n}$ a slowly varying smooth field, (16) spells out the assumption of finite-range antiferromagnetic order. We should stress that $\boldsymbol{n}$ and $\boldsymbol{\ell}$ are taken to be independent fields, constrained only by the orthogonality condition. This implies a doubling of degrees of freedom, which in principle should be corrected for when regularizing the theory. However, for our present purpose, to pinpoint the leading instabilities of the stripe electron dynamics due to the interaction with the antiferromagnetic domains, this issue is immaterial. The normalization of $\boldsymbol{\Omega}$ in (16) is only preserved up to $\mathcal{O}\left(a_{0}^{2}\right)$, but this is sufficient since we are interested in the long-wave length limit. In this limit we let the lattice spacing $a_{0}$ in the $x$-direction (parallel to the stripe) go to zero, expand all terms in the action $S$ which contain the spin fields up to $\mathcal{O}\left(a_{0}^{2}\right)$, and then do the replacements $a_{0} \sum_{r} \rightarrow \int d x, \boldsymbol{n}_{\boldsymbol{r}_{i}}(\tau) \rightarrow \boldsymbol{n}_{j^{(i)}}(\tau, x)$, and $\boldsymbol{\ell}_{\boldsymbol{r}_{i}}(\tau) \rightarrow \boldsymbol{\ell}_{j^{(i)}}(\tau, x)$. The result is a field theory for the independent orthogonal fields $\boldsymbol{n}$ and $\boldsymbol{\ell}$, with an action 


$$
\begin{aligned}
\mathcal{S}_{i}[\boldsymbol{n}, \boldsymbol{\ell}] & =\frac{S^{2} J_{H}}{2} \sum_{j^{(i)}} \int d x \int_{0}^{\infty} d \tau\left[\frac{1}{a_{0}}\left(\boldsymbol{n}_{j^{(i)}+1}(\tau, x)-\boldsymbol{n}_{j^{(i)}}(\tau, x)\right)^{2}+8 a_{0} \ell_{j^{(i)}}^{2}(\tau, x)\right] \\
& -i S \sum_{j^{(i)}} \int d x \int_{0}^{\infty} d \tau\left(\boldsymbol{n}_{j^{(i)}}(\tau, x) \times \partial_{\tau} \boldsymbol{n}_{j^{(i)}}(\tau, x)\right) \cdot \boldsymbol{\ell}_{j^{(i)}}(\tau, x)+\mathcal{S}_{i, B e r r y}[\boldsymbol{n}], \quad i=A, B,
\end{aligned}
$$

with

$\mathcal{S}_{i, B e r r y}[\boldsymbol{n}]=i S \sum_{\boldsymbol{r}_{i}} \gamma_{\boldsymbol{r}_{i}}(-1)^{\delta_{i B}} \int_{0}^{\infty} d \tau \boldsymbol{A}\left(\tau, \boldsymbol{r}_{i}\right) \cdot \partial_{\tau} \boldsymbol{n}\left(\tau, \boldsymbol{r}_{i}\right)$.

In the standard approach to the $2 \mathrm{D}$ antiferromagnet ${ }^{53}$ one would now integrate out the rapidly fluctuating $\boldsymbol{\ell}$ field from (17). After taking a continuum limit in the $y$-direction this would produce the familiar nonlinear $\sigma$ model $(\mathrm{NL} \sigma \mathrm{M})$, with an added sum over Berry phases, describing the slow long-wavelength dynamics of the Néel order-parameter field $\boldsymbol{n}$. In the present case, however, the localized spins adjacent to the stripe enter also in the Kondo lattice interaction (4), and this must be taken into account before one attempts to integrate out the $\boldsymbol{\ell}$-field. This problem is addressed next.

\section{Kondo Lattice Interaction: Mean-Field Decoupling}

Using (8) to write the electron spin density in (4) in terms of the chiral fields, and then replacing the spin operators $\boldsymbol{S}_{r, j^{(i)}}$ in (4) by the corresponding vectors $S \boldsymbol{\Omega}_{r, j^{(i)}}$, decomposing these as in (16), we obtain, expanding to $\mathcal{O}\left(a_{0}^{2}\right)$,

$$
H_{\text {Kondo }}=H_{\ell}+H_{\boldsymbol{n}}
$$

where

$$
\begin{array}{r}
H_{\boldsymbol{\ell}}=J_{K} S a_{0}^{2} \sum_{r} \boldsymbol{\Lambda}_{r} \cdot\left(\boldsymbol{\ell}_{r}^{(A)}+\boldsymbol{\ell}_{r}^{(B)}\right), \\
H_{\boldsymbol{n}}=J_{K} S a_{0} \sum_{r}(-1)^{r} \boldsymbol{\Lambda}_{r} \cdot\left(\boldsymbol{n}_{r}^{(A)}+\boldsymbol{n}_{r}^{(B)}\right),
\end{array}
$$

with

$$
\begin{aligned}
\boldsymbol{\Lambda}_{r} & =\left[\mathrm{e}^{2 i k_{F} r a_{0}} L_{r, \alpha}^{\dagger} R_{r, \beta}+\mathrm{e}^{-2 i k_{F} r a_{0}} R_{r, \alpha}^{\dagger} L_{r, \beta}\right. \\
& \left.+L_{r, \alpha}^{\dagger} L_{r, \beta}+R_{r, \alpha}^{\dagger} R_{r, \beta}\right] \boldsymbol{\sigma}_{\alpha \beta},
\end{aligned}
$$

measuring the spin density on the stripe. We have here used the notation introduced after Eq. (4), implying that $\boldsymbol{\ell}_{r}^{(i)} \equiv \boldsymbol{\ell}_{r, j^{(i)}=1}$ and $\boldsymbol{n}_{r}^{(i)} \equiv \boldsymbol{n}_{r, j^{(i)}=1}$. By the assumption that the Néel order directions of the $A$ and $B$ domains are shifted by $\pi$ relative to each other it follows that $\boldsymbol{n}_{r}^{(A)}=-\boldsymbol{n}_{r}^{(B)}$, and thus $H \boldsymbol{n}$ vanishes. It is here important to realize that there is no relative staggering of the ferromagnetic $A$ and $B$ components in (20). These rapidly fluctuating fields are independent, with no correlations across the stripe. This leaves us with $H_{\ell}$, which in the continuum limit, using (10) with $\boldsymbol{J} \equiv \boldsymbol{J}_{L}+\boldsymbol{J}_{R}$, takes the form

$$
H_{\boldsymbol{\ell}} \rightarrow 2 J_{K} S a_{0} \int d x \boldsymbol{J}(x) \cdot\left(\boldsymbol{\ell}^{(A)}(x)+\boldsymbol{\ell}^{(B)}(x)\right) .
$$

We have here dropped the nonchiral terms that mix $L$ and $R$-fields since these are washed out by the rapid phase oscillations in (22).

The fact that the stripe electrons couple manifestly only to the fast ferromagnetic components of the localized spins - independent of whether the stripe is metallic (Hubbard band away from half-filling) or insulating (half-filled band) ${ }^{58}$ - does not mean that the Néel order parameter dynamics is completely decoupled from the stripe electrons. The Néel field re-enters the problem via the orthogonality condition $\boldsymbol{n} \cdot \boldsymbol{\ell}=0$ which constrains the ferromagnetic component to a plane that follows its slow and smooth fluctuations. As we shall see next, part of the interaction in (23) can be absorbed as an effective spin density interaction among the stripe electrons. Since at low energies the Néel order direction is essentially constant over large patches in Euclidean space-time, this interaction will effectively be pinned in spin space, and hence break the spin rotational invariance of the electron spin dynamics on the relevant time- and length scales. This symmetry-breaking effect, driven by the Néel order in the environment, will dramatically influence the correlations of the stripe electrons.

For simplicity, we now treat the $A$ and $B$ domains separately. Starting with $A$, and collecting all terms in the action containing the $\boldsymbol{\ell}^{(A)}$-field defined on the $j^{(A)}=$ 1 spin array that couples to the stripe, we find from (17) and (23) the contribution to the partition function:

$$
Z_{\ell}^{(A)}=\int \mathcal{D}\left[\ell^{(A)}\right] \mathrm{e}^{-\mathcal{S}\left[\ell^{(A)}\right]},
$$

with 


$$
\mathcal{S}\left[\boldsymbol{\ell}^{(A)}\right]=\int_{0}^{\infty} d \tau \int d x\left[4 J_{H} a_{0} S^{2}\left(\boldsymbol{\ell}^{(A)}\right)^{2}+2 J_{K} S a_{0}\left(\boldsymbol{J}_{L}+\boldsymbol{J}_{R}\right) \cdot \boldsymbol{\ell}^{(A)}-i S\left(\boldsymbol{n}^{(A)} \times \partial_{\tau} \boldsymbol{n}^{(A)}\right) \cdot \boldsymbol{\ell}^{(A)}\right] .
$$

Note that we have again changed to the compact notation $\boldsymbol{n}_{j^{(A)}=1} \rightarrow \boldsymbol{n}^{(A)}, \boldsymbol{\ell}_{j(A)=1} \rightarrow \boldsymbol{\ell}^{(A)}$, introduced in Eqs. (20) and (21). The integral in (24) is Gaussian and can easily be carried out. We obtain

$$
\begin{gathered}
\int \mathcal{D}\left[\boldsymbol{\ell}^{(A)}\right] \exp \left(-\int d \tau d x\left[\left(\boldsymbol{\ell}^{(A)}\right)^{T} \boldsymbol{\Gamma} \boldsymbol{\ell}^{(A)}+\boldsymbol{\omega} \boldsymbol{\ell}^{(A)}\right]\right) \\
=\exp \left(\frac{1}{4} \int d \tau d x(\boldsymbol{\omega})^{T} \boldsymbol{\Gamma}^{-1} \boldsymbol{\omega}\right) \equiv \exp \left(-\mathcal{S}_{\ell^{(A)}}^{e f f}\right),
\end{gathered}
$$

where $\boldsymbol{\Gamma}=4 J_{H} a_{0} S^{2} \mathbf{1}, \boldsymbol{\omega}=\left(2 J_{K} S a_{0}\right) \boldsymbol{J}_{\perp}-i S\left(\boldsymbol{n}^{(A)} \times\right.$ $\left.\partial_{\tau} \boldsymbol{n}^{(A)}\right)$. We have here defined $\boldsymbol{J}_{\perp} \equiv \boldsymbol{J}-\left(\boldsymbol{J} \cdot \boldsymbol{n}^{(A)}\right) \boldsymbol{n}^{(A)}$, with $\boldsymbol{J} \equiv \boldsymbol{J}_{L}+\boldsymbol{J}_{R}$, as the piece of the electron spin density that - via the constraint $\boldsymbol{n}^{(A)} \cdot \boldsymbol{\ell}^{(A)}=0$ - survives the projection onto $\ell^{(A)}$. Thus, from (26) we obtain the effective action coming from fluctuations in $\ell^{(A)}$ :

$$
\mathcal{S}_{\boldsymbol{\ell}^{(A)}}^{e f f}=\int_{0}^{\infty} d \tau \int d x\left[-\frac{a_{0} J_{K}^{2}}{4 J_{H}} \boldsymbol{J}_{\perp} \cdot \boldsymbol{J}_{\perp}+\frac{i J_{K}}{8 \pi J_{H}}\left(\boldsymbol{n}^{(A)} \times \partial_{\tau} \boldsymbol{n}^{(A)}\right) \cdot \boldsymbol{J}_{\perp}+\frac{1}{16 J_{H} a_{0}}\left(\boldsymbol{n}^{(A)} \times \partial_{\tau} \boldsymbol{n}^{(A)}\right)^{2}\right]
$$

Let us in turn discuss the different contributions to $\mathcal{S}_{\ell^{(A)}}^{\text {eff }}$ :

First term in 27). The first term is an anisotropic spin interaction among the stripe electrons, induced by the coupling to the Néel-ordered spins in the $A$-domain. As we have already noted, this interaction follows the slow fluctuations of the $\boldsymbol{n}^{(A)}$-field along the stripe, with $\boldsymbol{J}_{\perp}$ constrained to a plane orthogonal to $\boldsymbol{n}^{(A)}$. To make progress we shall treat $\boldsymbol{n}^{(A)}$ in a mean-field formulation, and take it to be in a fixed (but arbitrary) direction $\langle\tilde{\boldsymbol{n}}\rangle$, defined by the antiferromagnetic order in the $A$-domain. Introducing a coordinate system $(x, y, z)$ with $\hat{z}$ in the direction of $\langle\tilde{\boldsymbol{n}}\rangle$, and using the operator identity $J_{L / R}^{z} J_{L / R}^{z}=\frac{1}{3} \boldsymbol{J}_{L / R} \cdot \boldsymbol{J}_{L / R}$, valid for chiral bilinears, the first term in (27) is then seen to add the interaction

$H_{\text {int }}=-\frac{a_{0} J_{K}^{2}}{2 J_{H}} \int d x\left(: J_{L}^{z} J_{L}^{z}:+: J_{R}^{z} J_{R}^{z}:+J_{L}^{x} J_{R}^{x}+J_{L}^{y} J_{R}^{y}\right)$

to the spin-sector stripe Hamiltonian in (12). The terms diagonal in $L$ and $R$ in (28) are forward scattering terms which renormalize the effective spin velocity $\tilde{v}_{s}$ on the stripe, $\tilde{v}_{s} \rightarrow \tilde{v}_{s}^{(A)}=\tilde{v}_{s}-a_{0} J_{K}^{2} /\left(4 \pi J_{H}\right)$, while the terms mixing $L$ - and $R$-fields describe backscattering of electrons and hence, when added to (12), shifts the corresponding coupling: $\tilde{g}_{0 \perp} \rightarrow \tilde{g}_{\perp}+a_{0} J_{K}^{2} /\left(4 \pi \tilde{v}_{s}^{(A)} J_{H}\right)$.

Second term in (27). Let us first recall54 that $\boldsymbol{n}^{(A)} \times \partial_{\tau} \boldsymbol{n}^{(A)}$ is an angular momentum density, which, at the extremum of the action in (17) is locked to the ferromagnetic component: $\boldsymbol{n}^{(A)} \times \partial_{\tau} \boldsymbol{n}^{(A)} \sim \boldsymbol{\ell}^{(A)}$. Since $\ell^{(A)}$ is a rapidly fluctuating field, the second term in (27), being a pure phase, for this case averages to zero already on finite patches in Euclidean space-time, and will be ignored in the low-energy limit considered here. This amounts to neglect fluctuations away from the cluster of paths that dominate the action (17) for the localized spins when decoupled from the stripe. As $J_{K} \ll J_{H}$, we do not expect these paths to change much when inserting the stripe, and the argument applies also in the presence of the stripe. We shall discuss the limitations of this mean-field type argument below.

Third term in (27). The last term in (27), containing only the Néel field and its time derivative, should be assembled with the spin action in (17). Then, integrating out all $\boldsymbol{\ell}_{j(A)}$ - fields from (5) - in exact analogy with the one-dimensional treatment of $\boldsymbol{\ell}_{j^{(A)}=1}$ in (26) taking a continuum limit in the $y$-direction, and using $\left(\boldsymbol{n} \times \partial_{\tau} \boldsymbol{n}\right)^{2}=\left(\partial_{\tau} \boldsymbol{n}\right)^{2}$, one obtains the effective action for the order parameter field in the $A$-domain:

$$
\begin{aligned}
\mathcal{S}[\boldsymbol{n}] & =\frac{1}{2 g_{0}} \int_{0}^{\infty} d \tau \int_{-\infty}^{\infty} d x \int_{0}^{\infty} d y\left[c(\nabla \mathbf{n})^{2}+\frac{1}{c}\left(\frac{\partial \mathbf{n}}{\partial \tau}\right)^{2}\right] \\
& +S_{\text {phase }}[\mathbf{n}],
\end{aligned}
$$

where the first term is the action for a nonlinear $\sigma$ model $(\mathrm{NL} \sigma \mathrm{M})$ in a semi-infinite plane, with parameters $g_{0}^{-1}=S / \sqrt{8} a_{0}, c=\sqrt{8} J S a_{0} \underline{54}$. One piece of the original sum over Berry phases in (17) has been absorbed in the $\mathrm{NL} \sigma \mathrm{M}$, while the part (18) containing the Néel field only, is left as a global phase $S_{\text {phase }}[\boldsymbol{n}]$ in (29). This phase is an alternating sum over the solid angles $\Phi\left[\boldsymbol{n}\left(\boldsymbol{r}_{i}, \tau\right)\right]$ swept by the local $\boldsymbol{n}\left(\boldsymbol{r}_{i}, \tau\right)$-fields as $\tau$ goes from 0 to $\infty$. As long as there are no disordering or finite-size effects causing discontinuities in the Néel field, 
$S_{\text {phase }}[\boldsymbol{n}]$ will be averaged out ${ }^{59}$. For this reason we will ignore it for the moment. For the more realistic case of a finite-width antiferromagnetic domain, modeled, say, by a spin ladder ${ }^{42}, S_{\text {phase }}[\boldsymbol{n}]$ will come into play, requiring a more careful analysis. We shall return to this important issue in Sec. III.D.

The analysis carried out for the $A$-domain above can be repeated step by step for the $B$-domain, and the fluc- tuations in $\ell^{(B)}$ are seen to give a contribution identical to that in (27), with the index " $A$ " replaced by " $B$ ". Summing the contributions from the two domains, it follows that the stripe electrons get described by an effective low-energy Hamiltonian

$$
H_{\text {stripe }}=H_{c}+H_{s}
$$

with $H_{c}$ defined in Eq. (11), while

$$
H_{s}=2 \pi v_{s} \int \mathrm{d} x\left\{: J_{L}^{z} J_{L}^{z}:+: J_{R}^{z} J_{R}^{z}:-g_{0 s} J_{L}^{z} J_{R}^{z}-g_{0 \perp}\left(J_{L}^{x} J_{R}^{x}+J_{L}^{y} J_{R}^{y}\right)\right\}
$$

where

$$
\begin{aligned}
g_{0 s} & =\frac{a_{0}}{\pi v_{F}}(U-2 V), \\
g_{0 \perp} & =\frac{a_{0}}{\pi v_{F}}\left(U-2 V+\frac{J_{K}^{2}}{2 J_{H}}\right), \\
v_{s} & =\tilde{v}_{s}-\frac{a_{0}}{2 \pi} \frac{J_{K}^{2}}{J_{H}}
\end{aligned}
$$

with $\tilde{v}_{s}$ defined in Eq. (13).

Thus, in contrast to the charge degrees of freedom which remain untouched by the coupling to the antiferromagnetic environment, the spin dynamics on the stripe is strongly renormalized by this same interaction and gets controlled by an effective U(1)-symmetric spin Hamiltonian (31). As for the low-energy processes in the decoupled antiferromagnetic environment, these are described by two independent NL $\sigma$ models, one for each domain $i=A, B$, as defined in (29). (It may be worthwhile pointing out that as long as the Néel field is protected by the low-energy thermodynamic limit, the phase $S_{\text {phase }}[\boldsymbol{n}]$ in (29) remains inactive ${ }^{59}$.)

\section{B. Diagonal Stripes}

The construction of the low-energy theory for a stripe running along the diagonal of the square lattice (FIG. 2) closely parallels that for a collinear stripe in the previous section.

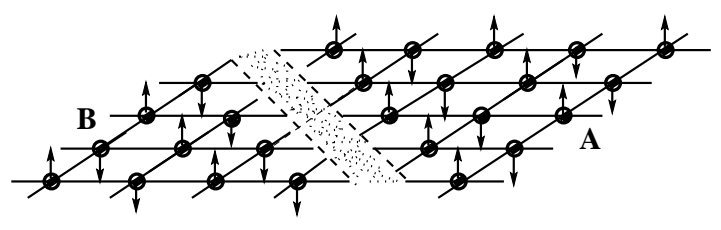

FIG. 2: Diagonal stripe structure.
We again model the isolated stripe by an extended $U-V$ Hubbard chain, but with a new hopping matrix element $t^{\prime}<t$ (since the overlaps between tight-binding orbitals along the diagonal of a lattice plaquette is expected to be smaller than along the bonds). As a result, the continuum theory in (11) and (12) still applies, but with $t$ replaced by $t^{\prime}$ (implying a shift of the effective velocities defined in (13)). As for the antiferromagnetic domains, we expect that the order parameter dynamics is still described by the NL $\sigma \mathrm{M}(29)$ in the bulk (away from the stripe): By assumption, the coupling to the stripe is weak, $J_{K} \ll J_{H}$, and can only perturb spins in its immediate neighborhood. Here we are interested in the reverse effect, and will explore how the Kondo lattice interaction (44) affects the electron dynamics on the diagonal stripe.

For this purpose, let us isolate one array of localized spins adjacent to the stripe, say the $A$-domain. We label the corresponding spin operators $\boldsymbol{S}_{r}^{(A)}$, where $r$ is a lattice coordinate running along the stripe that labels also the horizontal lattice axes that pierce the stripe at the corresponding sites.

The $\boldsymbol{S}_{r}^{(A)}$ spins interact with their neighboring spins on the parallel array, $\boldsymbol{S}_{r}^{(\tilde{A})}$ call them, via the terms

$$
H_{\text {array }}=J_{H} \sum_{j}\left(\boldsymbol{S}_{r}^{(A)} \cdot \boldsymbol{S}_{r}^{(\tilde{A})}+\boldsymbol{S}_{r-1}^{(A)} \cdot \boldsymbol{S}_{r}^{(\tilde{A})}\right)
$$

It is clear from the geometry that this interaction will generate an effective ferromagnetic coupling between the nearest-neighbor $\boldsymbol{S}^{(A)}$-spins, induced by a doubleexchange via the two neighboring $\boldsymbol{S}^{(\tilde{A})}$-spins. As seen in FIG. 2, this means that the local 1D magnetic environment sampled by the stripe electrons via the Kondo exchange is ferromagnetically ordered. Does this imply a different induced interaction among the stripe electrons as compared to the collinear case in (31)? To find out, let us first write down the full Kondo lattice interaction for the diagonal stripe, including the neighboring array 
of $\boldsymbol{S}_{r}^{(B)}$-spins from the $B$-domain:

$$
\begin{array}{r}
H_{\text {Kondo }} \\
=J_{K} \sum_{r, \alpha} c_{r, \alpha}^{\dagger} \boldsymbol{\sigma}_{\alpha \beta} c_{r, \beta} \cdot\left(\boldsymbol{S}_{r}^{(A)}+\boldsymbol{S}_{r+1}^{(A)}+\boldsymbol{S}_{r}^{(B)}+\boldsymbol{S}_{r-1}^{(B)}\right), \\
J_{K}>0 .
\end{array}
$$

Following the same route as in Sec II.A.3, using the decompositions (8) and (16), we obtain:

$$
H_{\text {Kondo }}=H_{\boldsymbol{\ell}}+H_{\boldsymbol{n}},
$$

where

$$
\begin{aligned}
& H_{\boldsymbol{\ell}}=J_{K} S a_{0}^{2} \sum_{r} \boldsymbol{\Lambda}_{r} \cdot\left(\boldsymbol{\ell}_{r}^{(A)}+\boldsymbol{\ell}_{r+1}^{(A)}+\boldsymbol{\ell}_{r}^{(B)}+\boldsymbol{\ell}_{r-1}^{(B)}\right), \\
& H_{\boldsymbol{n}}=J_{K} S a_{0} \sum_{r} \boldsymbol{\Lambda}_{r} \cdot\left(\boldsymbol{n}_{r}^{(A)}+\boldsymbol{n}_{r+1}^{(A)}+\boldsymbol{n}_{r}^{(B)}+\boldsymbol{n}_{r-1}^{(B)}\right),
\end{aligned}
$$

with $\boldsymbol{\Lambda}_{r}$ the local spin density operator on the stripe defined in (22). Similar to the collinear stripe treated above, the $\pi$-phase shifted Néel order across the diagonal stripe - along horizontal as well as vertical directions implies that $\boldsymbol{n}_{r}^{(A)}=-\boldsymbol{n}_{r}^{(B)}, \boldsymbol{n}_{r+1}^{(A)}=-\boldsymbol{n}_{r-1}^{(B)}$ (see FIG. 2), and it follows that $\mathrm{H}_{\boldsymbol{n}}$ in (37) vanishes, independent of the value of the stripe band filling $n_{e} / 2^{58}$. (Note that, in contrast to the collinear case in (21), there is no staggering factor in Eq. (37): the local magnetic environment as seen from the stripe is uniform along the stripe.) We are thus left with $\mathrm{H}_{\ell}$ in (36). Taking a continuum limit

$$
a_{0} \sum_{r} \rightarrow \int d x d y \delta(x-y),
$$

and doing a gradient expansion to $\mathcal{O}\left(a_{0}^{2}\right)$, we obtain

$$
\begin{array}{r}
H_{\boldsymbol{\ell}} \rightarrow \\
4 J_{K} S a_{0} \int d x \boldsymbol{J}(x, x) \cdot\left(\boldsymbol{\ell}^{(A)}(x, x)+\boldsymbol{\ell}^{(B)}(x, x)\right), \\
\boldsymbol{J}(x, x) \equiv \boldsymbol{J}_{L}(r)+\boldsymbol{J}_{R}(r),
\end{array}
$$

with the "diagonal" stripe coordinate $r$ replacing (the implicitly defined variable) $x$ in the definition of $\boldsymbol{J}_{L / R}$ after Eq. (23). Given (39), we now again focus on the $A$ domain, and isolate the piece of (39) containing only the $\ell^{(A)}$-field, $H_{\ell^{(A)}}$ call it. Inserting a time-dependence and letting $H_{\ell^{(A)}}$ act at (imaginary) time slice $\tau$, integrating over the slices, and adding the result to the ordinary semi-classical spin action $\mathcal{S}_{A}[\boldsymbol{n}, \boldsymbol{\ell}]$ for the $A$-domain (cf. with Eq. (6) for the corresponding collinear case), we obtain

$$
\begin{aligned}
& \mathcal{S}_{A}[\boldsymbol{n}, \boldsymbol{\ell}]+\int_{0}^{\infty} d \tau H_{\boldsymbol{\ell}} \\
& =\int_{-\infty}^{\infty} d x \int_{x}^{\infty} d y \int_{0}^{\infty} d \tau\left[\frac{J_{H} S^{2}}{2}\left(\left(\nabla \mathbf{n}^{(A)}\right)^{2}+8\left(\boldsymbol{\ell}^{(A)}\right)^{2}-i S\left(\boldsymbol{n}^{(A)} \times \partial_{\tau} \boldsymbol{n}^{(A)}\right) \cdot \boldsymbol{\ell}^{(A)}\right)+4 J_{K} S a_{0} \boldsymbol{J} \cdot \boldsymbol{\ell}^{(A)} \delta(x-y)\right] .
\end{aligned}
$$

Writing down the full $A$-domain action in (40) we have ignored the presence of the sum over Berry phases (cf. again Eq. (6) for the corresponding collinear case), since it does not involve the $\boldsymbol{\ell}$-field and hence does not couple directly to the stripe electrons. Also note that compared to our treatment of the collinear case in Sec. II.A.3 we have here shortcut the analysis by taking a continuum limit in the $y$-direction before integrating out the $\boldsymbol{\ell}$-field in (40). Carrying out the integration we obtain an effective $A$-domain action $\mathcal{S}_{\ell^{(A)}}^{e f f}$ generated by fluctuations in the ferromagnetic components of the localized spins:

$$
\mathcal{S}_{\boldsymbol{\ell}^{(A)}}^{e f f}=\int_{-\infty}^{\infty} d x \int_{x}^{\infty} d y \int_{0}^{\infty} d \tau\left[\left(-\frac{a_{0} J_{K}^{2}}{J_{H}} \boldsymbol{J}_{\perp} \cdot \boldsymbol{J}_{\perp}+\frac{i J_{K}}{2 J_{H}}\left(\boldsymbol{n} \times \partial_{\tau} \boldsymbol{n}\right) \cdot \boldsymbol{J}_{\perp}\right) \delta(x-y)+\frac{1}{16 J_{H}}\left(\boldsymbol{n} \times \partial_{\tau} \boldsymbol{n}\right)^{2}\right]
$$

with $\boldsymbol{J}_{\perp}$ defined after Eq. (26). Doing a saddle-point approximation and dropping the rapidly oscillating phase in (41), we obtain - in exact analogy with the collinear 
case - the induced stripe-electron interaction

$$
H_{i n t}=-\frac{2 a_{0} J_{K}^{2}}{J_{H}} \int d r\left(J_{L}^{x} J_{R}^{x}+J_{L}^{y} J_{R}^{y}+J_{L}^{z} J_{L}^{z}+J_{R}^{z} J_{R}^{z}\right),
$$

with $r$ the "diagonal" coordinate along the stripe.

This Hamiltonian is almost an exact copy of that for a collinear stripe, cf. Eq. (28), with the only difference that the magnitude of the coupling is larger by a factor of 4. (This can be traced back to the fact that on the lattice a diagonal stripe electron couples simultaneously to two localized spins in the $A$-domain.) Adding the identical contribution from the $B$-domain, it follows that the lowenergy spin dynamics on a diagonal stripe is described by the same effective Hamiltonian (31) as for the collinear case, but with the renormalized parameters in (32) modified by $J_{K} \rightarrow 2 J_{K}$. (As mentioned above, the hopping matrix element $t^{\prime}$ is also different from that which enters the free part of the Hamiltonian for a collinear stripe; cf. Eq. (30) with $v_{F}=2 a_{0} t \sin \left(\pi n_{e} / 2\right)$. However, as this has no impact on the problem studied here, from now on we use a single label $t$ for both types of stripes.)

In contrast to our analysis for the collinear stripe configuration, we have here not attempted to derive the full effective spin action for the decoupled environment. As we have already noted, away from the stripe the Néel order parameter dynamics is described by a NL $\sigma \mathrm{M}$ with an added Berry phase, as in (29). By inspection one finds that close to the diagonal stripe the Berry phase gets influenced by the unusual boundary condition associated with the diagonal stripe orientation. Thus, our results - here derived for a semi-infinite 2D geometry - may be of limited applicability for the case of "diagonal" finitewidth or "spin ladder" environments (see Sec. III.D). Their study is an interesting problem, but we here leave it for the future.

The fact that the same effective interaction appears for the diagonal and collinear stripe structures (up to the trivial $J_{K} \leftrightarrow 2 J_{K}$ shift) reflects its origin in the coupling of the electronic spin density to the uniform $\boldsymbol{\ell}$-components of the localized spins. These components are confined to a plane orthogonal to the Néel-direction, and are blind to whether the local Néel-field adjacent to a stripe is staggered (as for a collinear stripe) or uniform (diagonal stripe) ${ }^{60}$. The coupling constant $J_{K}^{2} / J_{H}$ embodies the second-order process that drives the induced interaction between the stripe electrons: An electron exchanges spin with the environment $\left(\sim J_{K}\right)$ and another electron arriving at the same lattice site flips back the localized spin by a second exchange $\left(\sim J_{K}\right)$, resulting in an effective spin exchange $\left(\sim J_{K}^{2}\right)$ between the two electrons. Since only that part of the spin exchange that couples to the $\boldsymbol{\ell}$-components survives, the effective interaction becomes anisotropic. The $1 / J_{H^{-}}$ dependence of the process is also expected: The larger the spin stiffness of the antiferromagnetic environment, the smaller the probability for the double exchange to occur.

\section{Effective Stripe Hamiltonian: Bosonization}

As we have seen, the low-energy electron dynamics on collinear as well as diagonal stripes - taking into account a weak spin exchange with the environment - is described by an effective Hamiltonian (30), with the amplitudes for forward- and backward scattering renormalized by the exchange. This Hamiltonian embodies a spin-anisotropic interaction among the electrons, well-defined on lengthand time scales over which the environment is magnetically ordered. To analyze the consequences for the stripe electron dynamics we shall use Abelian bosonization to map the model onto two independent quantum sineGordon models (in the weak-coupling limit)- one describing the collective charge excitations, the other the spin excitations - and then perform a renormalization group analysis to identify the leading instabilities of the system.

The method of bosonization is well reviewed in the literature 53.61 .62 , and we here only sketch the most important steps so as to fix notation and conventions. The standard bosonization formulas for spinful chiral electrons are given by 62 :

$$
\begin{aligned}
& R_{\alpha}(x)=\frac{1}{\sqrt{2 \pi a_{0}}} \eta_{\alpha} \mathrm{e}^{-i \sqrt{4 \pi} \phi_{R, \alpha}(x)}, \\
& R_{\alpha}^{\dagger}(x)=\frac{1}{\sqrt{2 \pi a_{0}}} \eta_{\alpha} \mathrm{e}^{i \sqrt{4 \pi} \phi_{R, \alpha}(x)}, \\
& L_{\alpha}(x)=\frac{1}{\sqrt{2 \pi a_{0}}} \bar{\eta}_{\alpha} \mathrm{e}^{i \sqrt{4 \pi} \phi_{L, \alpha}(x)}, \\
& L_{\alpha}^{\dagger}(x)=\frac{1}{\sqrt{2 \pi a_{0}}} \bar{\eta}_{\alpha} \mathrm{e}^{-i \sqrt{4 \pi} \phi_{L, \alpha}(x)},
\end{aligned}
$$

Here $\phi_{R, \alpha}(x)$ and $\phi_{L, \alpha}(x)$ are right- and left moving bosonic fields respectively, carrying spin $\alpha=\uparrow, \downarrow$. The Klein factors $\eta_{\alpha}$ and $\bar{\eta}_{\alpha}$ are inserted to ensure that the anticommutation relations for electron fields with different spin come out right. They are Hermitian and satisfy a Clifford algebra

$$
\left\{\eta_{\alpha}, \eta_{\beta}\right\}=\left\{\bar{\eta}_{\alpha}, \bar{\eta}_{\beta}\right\}=2 \delta_{\alpha \beta}, \quad\left\{\eta_{\alpha}, \bar{\eta}_{\beta}\right\}=0 .
$$

One next introduces charge $(c)$ and $\operatorname{spin}(s)$ fields $\varphi_{c, s}$ and their duals $\vartheta_{c, s}$ :

$$
\begin{array}{ll}
\varphi_{c}=\left(\phi_{\uparrow}+\phi_{\downarrow}\right) / \sqrt{2}, & \vartheta_{c}=\left(\theta_{\uparrow}+\theta_{\downarrow}\right) / \sqrt{2}, \\
\varphi_{s}=\left(\phi_{\uparrow}-\phi_{\downarrow}\right) / \sqrt{2}, & \vartheta_{s}=\left(\theta_{\uparrow}-\theta_{\downarrow}\right) / \sqrt{2},
\end{array}
$$

where

$$
\phi_{\alpha}=\phi_{L, \alpha}+\phi_{R, \alpha}, \quad \theta_{\alpha}=\phi_{L, \alpha}-\phi_{R, \alpha}
$$

Then, using the identities

$$
\begin{aligned}
& J_{R}+J_{L}=-(1 / \sqrt{\pi}) \partial_{x} \varphi_{c} \\
& J_{R}-J_{L}=-(1 / \sqrt{\pi}) \partial_{x} \vartheta_{c}
\end{aligned}
$$




$$
\begin{aligned}
J_{R}^{z}+J_{L}^{z} & =-(1 / \sqrt{2 \pi}) \partial_{x} \varphi_{s}, \\
J_{R}^{z}-J_{L}^{z} & =-(1 / \sqrt{2 \pi}) \partial_{x} \vartheta_{s},
\end{aligned}
$$

together with (43) and (44), we can translate $H_{\text {stripe }}=$ $H_{c}+H_{s}$ in (30) into bosonized form:

$$
\begin{gathered}
H_{c}=\frac{v_{c}}{2} \int d x\left\{\left(\partial_{x} \varphi_{c}^{\prime}\right)^{2}+\left(\partial_{x} \vartheta_{c}^{\prime}\right)^{2}+\delta_{1 n_{e}} \frac{2 m_{c}}{a_{0}^{2}} \kappa \cos \left(\sqrt{8 \pi K_{c}} \varphi_{c}^{\prime}\right)\right\} \\
H_{s}=\frac{v_{s}}{2} \int d x\left\{\left(\partial_{x} \varphi_{s}^{\prime}\right)^{2}+\left(\partial_{x} \vartheta_{s}^{\prime}\right)^{2}+\frac{2 m_{s}}{a_{0}^{2}} \kappa \cos \left(\sqrt{8 \pi K_{s}} \varphi_{s}^{\prime}\right)\right\} .
\end{gathered}
$$

We have here introduced the rescaled charge and spin fields

$$
\varphi_{c, s}^{\prime}=K_{c, s}^{-1 / 2} \varphi_{c, s}, \quad \vartheta_{c, s}^{\prime}=K_{c, s}^{1 / 2} \vartheta_{c, s},
$$

and the short-hand $\kappa \equiv \eta_{\uparrow} \eta_{\downarrow} \bar{\eta}_{\uparrow} \bar{\eta}_{\downarrow}$. To leading order in the coupling constants the sine-Gordon model parameters $K_{c(s)}$ and $m_{c(s)}$ are given by

$$
\begin{gathered}
2\left(K_{c}-1\right)=g_{0 c}=-\frac{a_{0}}{\pi v_{F}}(U+6 V), \\
2 \pi m_{c}=-g_{0 u}=-\frac{a_{0}}{\pi v_{F}}(U-2 V), \\
2\left(K_{s}-1\right)=g_{0 s}=\frac{a_{0}}{\pi v_{F}}(U-2 V), \\
2 \pi m_{s}=g_{0 \perp}=\frac{a_{0}}{\pi v_{F}}\left(U-2 V+\beta \frac{J_{K}^{2}}{J_{H}}\right),
\end{gathered}
$$

with

$$
\begin{aligned}
& v_{c}=v_{F}+\frac{a_{0}}{2 \pi}(U+6 V), \\
& v_{s}=v_{F}-\frac{a_{0}}{2 \pi}\left(U-2 V+2 \beta \frac{J_{K}^{2}}{J_{H}}\right),
\end{aligned}
$$

where

$$
\beta=\left\{\begin{array}{l}
1 / 2 \quad \text { collinear stripe } \\
2 \quad \text { diagonal stripe }
\end{array} .\right.
$$

Note that in obtaining (51) and (52), terms corresponding to scattering processes which lead to a renormalization of the Fermi velocities in second order in the couplings, as well as strongly irrelevant terms $\sim$ $\cos \left(\sqrt{8 \pi K_{c}} \varphi_{c}^{\prime}\right) \cos \left(\sqrt{8 \pi K_{s}} \varphi_{s}^{\prime}\right)$ describing Umklapp processes with parallel spins, have been omitted.

The product of Klein factors in (51) and (52) acts on a Hilbert space different from the boson Hilbert space and introduces a certain ambiguity into the formalism. We resolve it by choosing a representation of the Clifford algebra in terms of tensor products of Pauli matrices and the identity operator ${ }^{62}$,

$$
\begin{aligned}
& \eta_{\uparrow}=\sigma_{1} \otimes \sigma_{1}, \quad \eta_{\downarrow}=\sigma_{3} \otimes \sigma_{1} \\
& \bar{\eta}_{\uparrow}=\sigma_{2} \otimes \sigma_{1}, \quad \bar{\eta}_{\downarrow}=\mathbb{1} \otimes \sigma_{2},
\end{aligned}
$$

in which the above product $\kappa=\eta_{\uparrow} \eta_{\downarrow} \bar{\eta}_{\uparrow} \bar{\eta}_{\downarrow}$ of Klein factors has the form

$$
\kappa=\mathbb{1} \otimes \sigma_{3} .
$$

This matrix is diagonal with eigenvalues \pm 1 . Provided that all relevant correlation functions to be calculated contain only products of Klein factors which are simultaneously diagonal with $\kappa$ we can pick the eigenstate with eigenvalue +1 , say, and then ignore the rest of the Klein Hilbert space (allowing us to do the replacement $\kappa \rightarrow 1$ in (51) and (52)). We will come back to this point below.

\section{PAIRING AND DENSITY CORRELATIONS}

\section{A. Renormalization Group Analysis}

The mapping of the effective stripe Hamiltonian in Eq. (30) onto the quantum theory of two independent charge and spin Bose fields, manifestly shows that the collective low-energy charge- and spin dynamics on the stripe remains separated in the presence of a magnetic environment. This allows us to extract the ground state properties of the stripe electrons by performing independent renormalization group ( $\mathrm{RG}$ ) analyses of the chargeand spin sector sine-Gordon Hamiltonians. The RG flows are of Kosterlitz-Thouless type, with effective coupling constants $g_{i}(i=c, s, u, \perp)$, governed by the equations 63

$$
\begin{aligned}
d g_{c} / d \ell & =-g_{u}^{2}, \\
d g_{u} / d \ell & =-g_{c} g_{u},
\end{aligned}
$$

for the charge sector, and

$$
\begin{gathered}
d g_{s} / d \ell=-g_{\perp}^{2}, \\
d g_{\perp} / d \ell=-g_{s} g_{\perp},
\end{gathered}
$$

for the spin sector. Here $\ell=\ell n\left(a / a_{0}\right)$ with $a$ a renormalized length, while $g_{i}(\ell=0) \equiv g_{0 i}$ are the bare parameters that enter Eqs. (54) and (55). We shall denote by $\tilde{K}_{c(s)}$ and $\tilde{m}_{c(s)}$ the corresponding renormalized sine-Gordon parameters connected to $g_{i}$ via the same Eqs. (54) and (55). 
The flow lines lie on the hyperbolas

$$
g_{c(s)}^{2}-g_{u(\perp)}^{2}=g_{0 c(s)}^{2}-g_{0 u(\perp)}^{2},
$$

and - depending on the relation between $g_{0 c(s)}$ and $g_{0 u(\perp)}$ (or, equivalently, the bare sine-Gordon parameters $K_{c(s)}$ and $\left.m_{c(s)}\right)$ - exhibit two types of behaviors (cf. FIG. 3):

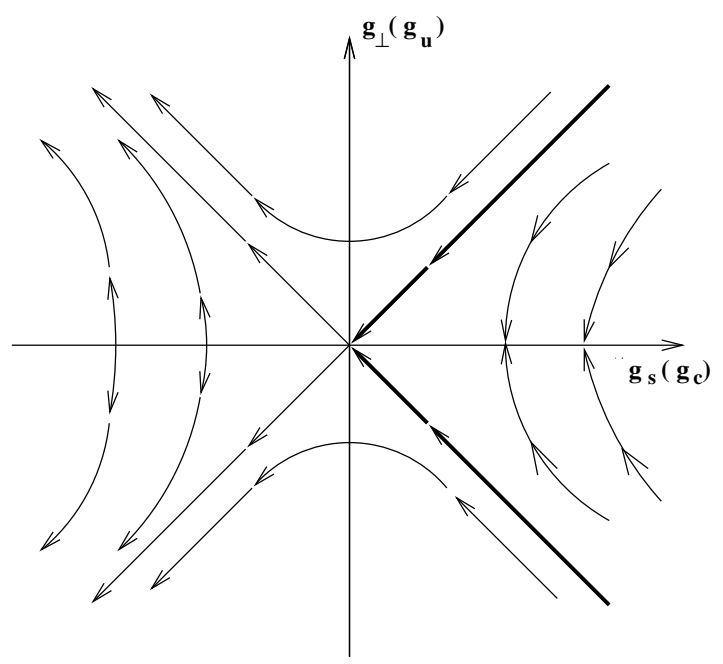

FIG. 3: Renormalization-group flow diagram for the spin $\left(g_{s}, g_{\perp}\right)$ and charge $\left(g_{c}, g_{u}\right)$ sectors. The arrows denote the direction of flow with increasing length scale.

Weak coupling regime.

When $g_{0 c(s)} \geq\left|g_{0 u(\perp)}\right|\left(K_{c(s)}-1 \geq \pi\left|m_{c(s)}\right|\right)$ we are in the weak coupling (Luttinger liquid) regime: $g_{u(\perp)} \rightarrow$ 0 , implying that the renormalized masses $\tilde{m}_{c(s)}$ scale to zero. The low-energy, long-wavelength behavior of the gapless charge (spin) degrees of freedom is thus described by a free scalar field

$$
H_{c(s)}=\frac{v_{c(s)}}{2} \int d x\left\{\left(\partial_{x} \varphi_{c(s)}^{\prime}\right)^{2}+\left(\partial_{x} \vartheta_{c(s)}^{\prime}\right)^{2}\right\} .
$$

Ignoring logarithmic corrections $\underline{64}$ coming from the slow renormalization of marginally irrelevant operators near the fixed line $g_{u(\perp)}=0$, the large-distance behaviors of the charge- and spin field correlators and their duals are given by

$$
\begin{gathered}
\left\langle\mathrm{e}^{i \sqrt{2 \pi K_{c(s)}^{*}} \varphi_{c(s)}^{\prime}(x)} \mathrm{e}^{-i \sqrt{2 \pi K_{c(s)}^{*}} \varphi_{c(s)}^{\prime}(0)}\right\rangle \sim|x|^{-K_{c(s)}^{*}}, 65 \\
\left\langle\mathrm{e}^{i \sqrt{2 \pi / K_{c(s)}^{*}} \vartheta_{c(s)}^{\prime}(x)} \mathrm{e}^{-i \sqrt{2 \pi / K_{c(s)}^{*}} \vartheta_{c(s)}^{\prime}(0)}\right\rangle \sim|x|^{-1 / K_{c(s)}^{*}} .
\end{gathered}
$$

Hence, the only parameters controlling the low-energy behavior in the gapless regimes are the fixed-point values $K_{c(s)}^{*}$ (Luttinger liquid parameters) of the renormalized coupling constants $\tilde{K}_{c(s)} \approx 1+g_{c(s)} / 2$.
Strong coupling regimes.

When $g_{0 c(s)}<\left|g_{0 u(\perp)}\right|\left(K_{c(s)}-1<\pi\left|m_{c(s)}\right|\right)$ the system scales to strong coupling. The two separatrices $g_{0 c(s)}= \pm\left|g_{0 u(\perp)}\right|$ divide the strong coupling regimes for charge and spin into two sectors respectively: (i) $g_{0 c(s)} \leq-\left|g_{0 u(\perp)}\right|$, where the increase of $\left|g_{c(s)}\right|$ and $\left|g_{u(\perp)}\right|$ is immediate, and (ii) $-\left|g_{0 u(\perp)}\right|<g_{0 c(s)}<\left|g_{0 u(\perp)}\right|$, where one observes a crossover from a weak-coupling behavior at intermediate scales $\left(g_{c(s)} \approx\left|g_{u(\perp)}\right|\right)$ to strong coupling at larger scales $\left(g_{c(s)} \approx-\left|g_{u(\perp)}\right|\right)^{61}$. Depending on the sign of the bare mass $m_{c(s)}$ in (54) and (55), the renormalized mass $\tilde{m}_{c(s)}$ is driven to $\pm \infty$, signaling a flow to one of the two strong coupling regimes, with a dynamical generation of a commensurability gap $\Delta_{c(s)}$ in the charge (spin) excitation spectrum. The flow of $\left|\tilde{m}_{c(s)}\right|$ to large values indicates that the cos-term in the sine-Gordon model dominates the large-distance properties of the charge (spin) sector. With the cosine-term being the dominant one, the values of $\varphi_{c(s)}^{\prime}$ will tend to be pinned at the minima of the cosine potential. For $m_{c(s)}<0$ these are at $\sqrt{8 \pi K_{c(s)}} \varphi_{c(s)}^{\prime}=2 \pi n$, with $n$ an arbitrary integer. Since $\varphi_{c(s)}^{\prime}$ are angular variables one cannot distinguish between different $n$, however, and the "negative mass" condensation is defined by $\left\langle\varphi_{c(s)}^{\prime}\right\rangle=0$. Similarly, for $m_{c(s)}>0$ the minima are at $\sqrt{8 \pi K_{c(s)}} \varphi_{c(s)}^{\prime}=\pi n$ and the fields order at $\sqrt{\pi / 8 K_{c(s)}}$. To summarize, there are two strong-coupling regimes where the fields $\varphi_{c(s)}^{\prime}$ get ordered with the expectation values

$$
\left\langle\varphi_{c(s)}^{\prime}\right\rangle=\left\{\begin{array}{ll}
\sqrt{\pi / 8 K_{c(s)}}, & m_{c(s)}>0 \\
0, & m_{c(s)}<0
\end{array} .\right.
$$

Note that the signs of the bare masses in (67) are contingent upon the choice of truncated Klein Hilbert space in Sec. II.C, where we have taken $\kappa \rightarrow 1$ in (51) and (52). This has no effect on the physics, however, since a transposition of the two strong-coupling phases above (via the alternative choice $\kappa \rightarrow-1$ ) would be followed by a subsequent redefinition of any relevant correlation function, thus producing the same value of any observable.

Having exposed the properties of the weak- and strong coupling regimes, let us apply the results first to the charge sector. By inspection of the "bare" values of the coupling constants in the charge sector, Eq. (54), one easily finds, using Eqs. (67), that for a half-filled band $\left(n_{e}=1\right)$ this sector is gapped for $U>2|V|$ and for $U<2 V$ when $V>0$ (strong coupling regimes). In the former case $\tilde{m}_{c} \rightarrow-\infty$, implying that

$$
\left\langle\varphi_{c}^{\prime}\right\rangle=0,
$$

while in the latter case $\tilde{m}_{c} \rightarrow \infty$, with

$$
\left\langle\varphi_{c}^{\prime}\right\rangle=\sqrt{\pi / 8 K_{c}} .
$$


For any other values of $U$ and $V$, but still at half-filling, we are in the weak-coupling regime, corresponding to a gapless charge excitation spectrum. The charge degrees of freedom are here governed by the free Bose field in Eq. (64), with the fixed-point value of the charge parameter

$$
K_{c}^{*} \simeq 1+\frac{2}{\pi v_{c}} \sqrt{V(U+2 V)}>1, \quad n_{e}=1,
$$

obtained from (63) with $\tilde{m}_{c}=0$. The line $U=2 \mathrm{~V}>0$ corresponding to the fixed-point line $m_{c}=0, K_{c}-1<0$, is special. Here the low-energy properties of the gapless charge sector are described by the free massless Bose field in (64) with $K_{c}^{*}=K_{c}$, reflecting its exact marginality.

Away from half-filling $\left(n_{e} \neq 1\right)$ the bare mass term in (51) is killed off for any values of $U$ and $V$, and the charge degrees of freedom are described by the free Bose field in (64). Analogous to the special line $U=2 \mathrm{~V}>0$ above, the correlations in (65) and (66) are now governed by the bare value of the Luttinger liquid charge parameter in (54):

$$
K_{c}^{*}=K_{c}=1-\frac{a_{0}}{2 \pi v_{c}}(U+6 V), \quad n_{e} \neq 1 .
$$

All of the above is familiar from conventional "gology" for Hubbard-type models ${ }^{12.55}$. Since the Kondo lattice interaction, Eq. (4), does not couple to the charge sector the latter indeed behaves as if the electrons were isolated from the magnetic environment.

Let us now look at the behavior of the spin sector, which is more interesting. As we have seen, the spin exchange between the Néel-ordered environment and the stripe electrons breaks the $S U(2)$ spin-rotational symmetry in the effective theory. This implies that the spin sector is gapped for arbitrary $U \neq 2 V-\beta J_{K}^{2} / J_{H}$ (strong coupling regime). When $U>2 V-\beta J_{K}^{2} / J_{H}$ the mass renormalization goes to $+\infty$, whereas for $U<$ $2 V-\beta J_{K}^{2} / J_{H}$ the mass renormalizes to $-\infty$. Reading off from (67), using (55), this implies the spin field orderings

$$
\left\langle\varphi_{s}^{\prime}\right\rangle=\left\{\begin{array}{ll}
\sqrt{\pi / 8 K_{s}}, & U>2 V-\beta J_{K}^{2} / J_{H} \\
0, & U<2 V-\beta J_{K}^{2} / J_{H}
\end{array} .\right.
$$

Note that this result is independent of the band filling on the stripe.

When $J_{K}=0$ and the stripe decouples from the environment, the $\mathrm{SU}(2)$ invariance of the spin sector is recovered, and the spin dynamics renormalizes along the separatrix $g_{s}=g_{\perp}$. As is well-known, the spin sector then gets controlled by the weak-coupling Luttinger liquid parameter $K_{s} \simeq 1+\frac{1}{2} g_{s} \rightarrow K_{s}^{*}=1$ when $U>2 V^{55}$, whereas for $U<2 \mathrm{~V}$ one stays in the strong coupling regime with $\left\langle\varphi_{s}^{\prime}\right\rangle=0^{65,66}$.

Next, we want to exploit the RG results derived above to map out the groundstate phase diagram for the stripe electrons. We shall catalogue the different phases according to the values of $\left(U, V, J_{K}^{2} / J_{H}, n_{e}\right)$, and focus on the corresponding behaviors of density and superconducting pairing fluctuations. These are characterized by the correlations of the associated order parameters, which in the present case come in two guises: (1) conventional and (2) composite order parameters. Let us in turn review their definitions and bosonized representations.

\section{B. Order Parameters}

\section{Conventional Order Parameters}

The conventional order parameters 55.61 .66 .67 which may develop long-range correlations in this class of models are those of short wavelength $\left(2 k_{F} x\right)$ fluctuations of the site- and bond-located charge density, site- and bond-located spin density and superconducting singlet and triplet pairing. By using the chiral decomposition (8) of the electron fields together with the bosonization dictionary in Eq. (43) - (50) it is straightforward to obtain their bosonized forms.

- Charge density wave.

This fluctuation is carried by the charge-0, spin-0 excitations created by the operator

$$
\begin{aligned}
& \mathcal{O}_{C D W}(r)=\sum_{\alpha} c_{r, \alpha}^{\dagger} c_{r, \alpha} \\
= & \sum_{\alpha}\left(J_{R, \alpha}(r)+J_{L, \alpha}(r)+\delta_{r} L_{\alpha}^{\dagger}(r) R_{\alpha}(r)+\delta_{r}^{-1} R_{\alpha}^{\dagger}(r) L_{\alpha}(r)\right)
\end{aligned}
$$

with $\delta_{r}=\mathrm{e}^{2 i k_{F} r a_{0}}$, and where $J_{L / R}$ are the chiral charge currents defined in Eq. (9). Keeping only the finitemomentum modes $\left(k= \pm 2 k_{F}\right)$ from the nonchiral terms, taking a continuum limit, and reading off from the dictionary (43)-(50), one obtains the bosonized expression

$$
\begin{aligned}
& \mathcal{O}_{C D W}(x) \rightarrow \\
& \quad \sin \left(\sqrt{2 \pi K_{c}} \varphi_{c}^{\prime}(x)-2 k_{F} x\right) \cos \left(\sqrt{2 \pi K_{s}} \varphi_{s}^{\prime}(x)\right),
\end{aligned}
$$

where we have used that $\bar{\eta}_{\uparrow} \eta_{\uparrow}$ and $\bar{\eta}_{\downarrow} \eta_{\downarrow}$ are diagonal with the same eigenvalue on the truncated Klein Hilbert space chosen in Sec II.C.

\section{- Spin-density wave.}

This is the simplest charge-0, spin-1 vector order parameter, and is defined by

$$
\boldsymbol{\mathcal { O }}_{S D W}(r)=\frac{1}{2} c_{r, \alpha}^{\dagger} \boldsymbol{\sigma}_{\alpha \beta} c_{r, \beta} .
$$

Bosonizing the $x$-component of $\mathcal{O}(n)_{S D W}$ that creates $k= \pm 2 k_{F}$ excitations, and dropping the trivial $k=0$ modes, one finds in the long-wave length continuum limit

$$
\begin{aligned}
& \mathcal{O}_{S D W}^{x}(x) \rightarrow \\
& \bar{\eta}_{\uparrow} \eta_{\downarrow} \cos \left(\sqrt{2 \pi K_{c}} \varphi_{c}^{\prime}(x)-2 k_{F} x\right) \sin \left(\sqrt{2 \pi K_{s}^{-1}} \vartheta_{s}^{\prime}(x)\right) .
\end{aligned}
$$


To obtain this form we have exploited the fact that the Klein factors $\bar{\eta}_{\uparrow} \eta_{\downarrow},-\bar{\eta}_{\downarrow} \eta_{\uparrow}, \eta_{\uparrow} \bar{\eta}_{\downarrow}$ and $-\eta_{\downarrow} \bar{\eta}_{\uparrow}$ have the same action in the truncated Klein Hilbert space. (Note, however, that $\bar{\eta}_{\uparrow} \eta_{\downarrow}$ is not diagonal on this space, and as a reminder of this we keep it explicitly in Eq. (76)). In the same way one easily obtains

$$
\begin{aligned}
& \mathcal{O}_{S D W}^{y}(x) \rightarrow \\
& \bar{\eta}_{\uparrow} \eta_{\downarrow} \cos \left(\sqrt{2 \pi K_{c}} \varphi_{c}^{\prime}(x)-2 k_{F} x\right) \cos \left(\sqrt{2 \pi K_{s}^{-1}} \vartheta_{s}^{\prime}(x)\right),
\end{aligned}
$$

and

$$
\begin{aligned}
& \mathcal{O}_{S D W}^{z}(x) \rightarrow \\
& \quad \cos \left(\sqrt{2 \pi K_{c}} \varphi_{c}^{\prime}(x)-2 k_{F} x\right) \sin \left(\sqrt{2 \pi K_{s}} \varphi_{s}^{\prime}(x)\right),
\end{aligned}
$$

where in the $z$-component we have put $\bar{\eta}_{\uparrow} \eta_{\uparrow}=\bar{\eta}_{\downarrow} \eta_{\downarrow}=$ constant.

In the special case of a half-filled band $\left(n_{e}=1\right)$ one can distinguish between the $2 k_{F}$ modulations of the charge and spin densities with extrema of the density profile located on sites or between sites - i.e. on bonds. Therefore at half-filling one should also consider order parameters corresponding to the short wavelength fluctuations of bond-located charge and spin densities:

- Bond-located charge density wave ("dimer").
A dimerization instability is characterized by enhanced correlations among the charge-0, spin-0 excitations created by

$$
\mathcal{O}_{b C D W}(r)=\sum_{\alpha}\left(c_{r, \alpha}^{\dagger} c_{r+1, \alpha}+\text { h.c. }\right) .
$$

Again, keeping only the $k= \pm 2 k_{F}$ excitations, one obtains in the continuum limit

$$
\begin{aligned}
& \mathcal{O}_{b C D W}(x) \rightarrow \\
& \quad \cos \left(\sqrt{2 \pi K_{c}} \varphi_{c}^{\prime}(x)-2 k_{F} x\right) \cos \left(\sqrt{2 \pi K_{s}} \varphi_{s}^{\prime}(x)\right) .
\end{aligned}
$$

- Bond-located spin-density wave.

This is the vector order parameter that describes charge-0, spin-1 magnetic excitations centered on the lattice bonds:

$$
\mathcal{O}_{b S D W}(r)=\frac{1}{2} \sum_{\alpha, \beta}\left(c_{r, \alpha}^{\dagger} \sigma_{\alpha \beta} c_{r+1, \beta}+\text { h.c. }\right) .
$$

In the continuum limit the lattice shift in (81) shows up as an extra phase $\pi / 2$ added to the ubiquitous phase $2 k_{F} x$ (cf. the bosonized dimer operator in (80)) and we thus identify the bosonized components of the finitemomentum part of $\mathcal{O}_{b S D W}(x)$ as

$$
\mathcal{O}_{b S D W}^{i}(x) \rightarrow \begin{cases}\bar{\eta}_{\uparrow} \eta_{\downarrow} \sin \left(\sqrt{2 \pi K_{c}} \varphi_{c}^{\prime}(x)-2 k_{F} x\right) \sin \left(\sqrt{2 \pi K_{s}^{-1}} \vartheta_{s}^{\prime}(x)\right), & i=x, \\ \bar{\eta}_{\uparrow} \eta_{\downarrow} \sin \left(\sqrt{2 \pi K_{c}} \varphi_{c}^{\prime}(x)-2 k_{F} x\right) \cos \left(\sqrt{2 \pi K_{s}^{-1}} \vartheta_{s}^{\prime}(x)\right), & i=y, \\ \sin \left(\sqrt{2 \pi K_{c}} \varphi_{c}^{\prime}(x)-2 k_{F} x\right) \sin \left(\sqrt{2 \pi K_{s}} \varphi_{s}^{\prime}(x)\right), & i=z\end{cases}
$$

Finally, we consider the two order parameters for (superconducting) pairing:

- Singlet pairing.

The charge-2e, spin-0 superconducting pairing modes on the stripe lattice are created by the operator

$$
\mathcal{O}_{S S}(r)=c_{r, \uparrow}^{\dagger} c_{r, \downarrow}^{\dagger}=\delta_{r} L_{\uparrow}^{\dagger}(r) L_{\downarrow}^{\dagger}(r)+\delta_{r}^{-1} R_{\uparrow}^{\dagger}(r) R_{\downarrow}^{\dagger}(r)+L_{\uparrow}^{\dagger}(r) R_{\downarrow}^{\dagger}(r)+R_{\uparrow}^{\dagger}(r) L_{\downarrow}^{\dagger}(r) .
$$

The $k= \pm 2 k_{F}$ excitations produced by the chiral terms are the so called $\eta$-pairing modes 68 . The right-moving $\eta$-pairs can be written as

$$
\eta_{R}(x) \equiv R_{\uparrow}^{\dagger}(x) R_{\downarrow}^{\dagger}(x) \rightarrow \exp \left(i \sqrt{2 \pi K_{c}^{-1}} \vartheta_{c}^{\prime}(x)\right) \exp \left(-i\left(\sqrt{2 \pi K_{c}} \varphi_{c}^{\prime}(x)-2 k_{F} x\right)\right)
$$

in the long-wave length limit, with the analogous expression for left-moving pairs, $\eta_{L}(x) \equiv L_{\uparrow}^{\dagger}(x) L_{\downarrow}^{\dagger}(x)$. As these contain only the charge field and its dual, they are blind to the antiferromagnetic environment and hence we will not consider them here. This leaves us with the $k=0$ BCS singlet pairing operator contained in (83), with the bosonized form

$$
\mathcal{O}_{S S}(x) \rightarrow \eta_{\uparrow} \bar{\eta}_{\downarrow} \exp \left(i \sqrt{2 \pi K_{c}^{-1}} \vartheta_{c}^{\prime}(x)\right) \cos \left(\sqrt{2 \pi K_{s}} \varphi_{s}^{\prime}(x)\right)
$$


- Triplet pairing.

The charge-2e, spin-1 pairing modes are created by the lattice operator

$$
\mathcal{O}_{T S}(r)=-i c_{r, \alpha}^{\dagger}\left(\boldsymbol{\sigma} \sigma^{y}\right)_{\alpha \beta} c_{r, \beta}^{\dagger} .
$$

Again retaining only the $k=0$ modes,

$$
\mathcal{O}_{T S}(r) \rightarrow-i R_{\alpha}^{\dagger}(r)\left(\boldsymbol{\sigma} \sigma^{y}\right)_{\alpha \beta} L_{\beta}^{\dagger}(r),
$$

we obtain for the bosonized components in the long-wave length limit:

$$
\mathcal{O}_{T S}^{i} \rightarrow \begin{cases}\exp \left(i \sqrt{2 \pi K_{c}^{-1}} \vartheta_{c}^{\prime}(x)\right) \sin \left(\sqrt{2 \pi K_{s}^{-1}} \vartheta_{s}^{\prime}(x)\right), & i=x, \\ \exp \left(i \sqrt{2 \pi K_{c}^{-1}} \vartheta_{c}^{\prime}(x)\right) \cos \left(\sqrt{2 \pi K_{s}^{-1}} \vartheta_{s}^{\prime}(x)\right), & i=y, \\ \eta_{\uparrow} \bar{\eta}_{\downarrow} \exp \left(i \sqrt{2 \pi K_{c}^{-1}} \vartheta_{c}^{\prime}(x)\right) \sin \left(\sqrt{2 \pi K_{s}} \varphi_{s}^{\prime}(x)\right), & i=z,\end{cases}
$$

\section{Composite Order Parameters}

In addition to the conventional order parameters listed above we need to consider composite order parameters built from operators acting on the stripe electrons and the magnetic environment. The notion of composite order parameters was first exploited in the theory of superconductivity 27,28 , where it was realized that since any product of a particle-hole (i.e. charge-neutral) operator and a Cooper pair operator possess charge $2 e$ this composite can, in principle, describe some superconducting state. By analogy, one may similarly construct composite CDW and SDW order parameters.

We shall here introduce only composite order parameters that may develop long-range correlations for the physically most interesting case of a stripe away from half-filling and with repulsive electron-electron interactions $U-2 V>0$ : composite CDW and composite singlet pairing.

\section{- Composite (site-located) charge density wave.}

A composite CDW order parameter is obtained by projecting the conventional (site-centered) spin- $1 / 2 \mathrm{SDW}$ onto the difference between the localized spins on the neighboring $A$ - and $B$-arrays:

$$
\mathcal{O}_{c-C D W} \sim \mathcal{O}_{S D W} \cdot\left(\boldsymbol{S}^{(A)}-\boldsymbol{S}^{(B)}\right) .
$$

Note that this expression is well-defined in the continuum limit for any stripe geometry: In particular, for the case of a diagonal structure, an electron at the $r^{t h}$ site on the stripe couples to $\boldsymbol{S}_{r-1}^{(A)}+\boldsymbol{S}_{r}^{(A)}$ in the $A$-domain, which in the continuum limit reduces to $2 \boldsymbol{S}^{(A)}\left(x_{r}\right)$, dropping an irrelevant gradient term. Considering first a collinear structure, we need to keep only the staggered parts $\boldsymbol{n}_{r}^{(A)}(-1)^{r}$ and $\boldsymbol{n}_{r}^{(B)}(-1)^{r}$ of the localized spins since the correlations of the uniform components of $\boldsymbol{S}_{r}^{(A)}$ and $\boldsymbol{S}_{r}^{(B)}$ die out fast ${ }^{53}$. With a phase-antiphase domain, as assumed here, we further have that $\boldsymbol{n}^{(A)}=-\boldsymbol{n}^{(B)} \equiv \boldsymbol{n}$.
Thus, from (89), the $k=2 k_{F}+\pi / a_{0} \equiv 2 k_{F}^{*}$ part of the composite charge density wave is given by

$$
\mathcal{O}_{c-C D W}^{\left(k=2 k_{F}^{*}\right)} \sim \mathcal{O}_{S D W} \cdot \boldsymbol{n}(-1)^{r},
$$

with $r$ the stripe lattice coordinate, and with the bosonized components of $\mathcal{O}_{S D W}$ written down in Eqs. (76) - (78). It would be tempting to refer to the generalized Luttinger theorem 69 to "explain" the appearance of the composite staggered CDW, Eq. (90). As pointed out by Zachar ${ }^{26}$, the theorem asserts that theories belonging to the class of Kondo-Heisenberg lattice type models are expected to support a massless spin-0, charge- 0 excitation of momentum $k=2 k_{F}^{*}$ (reflecting the presence of a "large Fermi surface" due to the localized spins). However, in the present case the localized spins of the environment are assumed to be ordered (with the $\mathrm{NL} \sigma \mathrm{M}$ in (29) describing the small fluctuations of the order parameter field $\boldsymbol{n}$ ), and, as a consequence, time reversal symmetry - entering as a condition for the validity of the theorem ${ }^{69}$ - is broken. Indeed, the case of a diagonal structure is different, and does not produce a $k=2 k_{F}^{*}$ mode. Here the stripe electrons experience a local ferromagnetic environment (cf. FIG. 2), and the composite CDW now appears at $k=2 k_{F}$ (i.e. with no staggering):

$$
\mathcal{O}_{c-C D W}^{\left(k=2 k_{F}\right)} \sim \mathcal{O}_{S D W} \cdot \boldsymbol{n} .
$$

\section{- Composite singlet pairing.}

By taking the product of the conventional triplet pairing operator $\mathcal{O}_{T S}$ for the stripe with the difference of spin operators for localized spins, $\boldsymbol{S}^{(A)}-\boldsymbol{S}^{(B)}$, a composite singlet pairing operator can be formed as

$$
\mathcal{O}_{c-S S} \sim \mathcal{O}_{T S} \cdot\left(\boldsymbol{S}^{(A)}-\boldsymbol{S}^{(B)}\right) .
$$

For a collinear stripe this operator has two momentum components: a uniform $k=0$ composite singlet

$$
\mathcal{O}_{T S} \cdot\left(\boldsymbol{\ell}^{(A)}-\boldsymbol{\ell}^{(B)}\right)
$$


with rapidly decaying correlations due to the incoherent fluctuations of $\ell^{(A)}$ and $\boldsymbol{\ell}^{(B)}$, and a $k=\pi / a_{0}$ staggered composite singlet

$$
\begin{aligned}
\mathcal{O}_{c-S S}^{\left(k=\pi / a_{0}\right)} & \sim \mathcal{O}_{T S} \cdot \boldsymbol{n}(-1)^{r} \\
& =-i R_{\alpha}^{\dagger}(r)\left(\boldsymbol{\sigma} \sigma^{y}\right)_{\alpha \beta} L_{\beta}^{\dagger}(r) \cdot \boldsymbol{n}(-1)^{r},
\end{aligned}
$$

with $r$ the discrete lattice coordinate along the stripe, and where $\boldsymbol{n} \equiv \boldsymbol{n}^{A}=-\boldsymbol{n}^{B}$. It is important to note that $\mathcal{O}_{c-S S}^{\left(k=\pi / a_{0}\right)}$ is odd under time reversal $(T: R \leftrightarrow L, \boldsymbol{\sigma} \rightarrow$ $-\boldsymbol{\sigma}, \boldsymbol{n} \rightarrow-\boldsymbol{n})$, as well as under parity $(P: R \leftrightarrow L)$, implying "odd-frequency odd-parity pairing" 29 .

Turning to the case of a diagonal stripe structure the composite singlet pairing now occurs for $k=0$ (since the local magnetic environment appears uniform as seen from the stripe), and one has

$$
\mathcal{O}_{c-S S}^{(k=0)} \sim-i R_{\alpha}^{\dagger}(r)\left(\boldsymbol{\sigma} \sigma^{y}\right)_{\alpha \beta} L_{\beta}^{\dagger}(r) \cdot \boldsymbol{n} .
$$

Again, parity and time reversal are broken. It is important to point out that theoretical work ${ }^{70}$ suggests that odd-frequency pairing is actually unstable for $k=0$ pairs (at least within Eliashberg-Migdal theory, where vertex corrections to the self-energy are neglected). This result becomes particularly intriguing when seen in the light of the diagonal $\rightarrow$ collinear stripe rotation associated with the superconducting transition observed in some of the cuprates 38.39 (cf. our discussion in Sec. I): If the singlet pairing in the high- $T_{c}$ materials were of composite nature, the stripe rotation would precisely serve to stabilize the pairing by shifting the momentum from $k=0$ (diagonal configuration with unstable pairing) to $k=\pi / a_{0}$ (collinear configuration with a stable, staggered composite pairing mode)!

In the presence of 2D Néel order, as assumed here, the $\boldsymbol{n}$-field correlations are infinitely ranged in the groundstate, and the $\mathcal{O}_{c-S S}^{k=\pi / a_{0}}$ and $\mathcal{O}_{c-S S}^{k=0}$ operators may build up large-distance correlations that compete effectively with conventional triplet pairing. Whether this happens, and what other order parameter correlations may develop, will be studied next. For an extended discussion of composite order parameters for 1D correlated electrons, we refer the reader to Refs. 26 and 28.

\section{Phases}

Equipped with the results in the two previous sections we shall now pinpoint the leading groundstate instabilities of the stripe electrons and list the corresponding phases:

\section{Half-filled band: $n_{e}=1$}

The phase diagram consists of five sectors: A, B, C, D1, and D2 (see FIG. 4).
- A phase: $U>2|V|$.

We include this case merely as an illustration of our formalism, as a half-filled band (one electron per site on the stripe) is somewhat special when combined with dominant repulsive on-site interaction. The reason is that one here expects to loose the phase-antiphase configuration as $n_{e} \rightarrow 1$, and instead recover the undoped antiferromagnetic state (with an in-phase Néel configuration across the "stripe"). In work by Zachar ${ }^{56}$, based on a stripe t-J model (corresponding to a "strong- coupling" limit $J_{K}=J_{H}$ of our lattice model in (10), it was suggested that there is a transition from the phase-antiphase to inphase Néel configuration already at a band filling $\sim 0.6$ (see also Ref. 57). Still, it is instructive to explore the assumption of a half-filled stripe embedded in a hypothetical phase-antiphase Néel background, and explore the consequences. At half-filling the charge excitation spectrum is gapped $\left(\Delta_{c} \neq 0\right)$ when $U>2|V|$. The stripe is thus insulating and the ordering of the charge boson with ground state expectation value $\left\langle\varphi_{c}^{\prime}\right\rangle=0$ suppresses the conventional CDW and superconducting correlations, but leaves behind the SDW and Peierls (dimerized) correlations. Turning to the spin sector, according to Eq. (72) there is a condensation at $\left\langle\varphi_{s}^{\prime}\right\rangle=\sqrt{\pi / 8 K_{s}}$. This kills off the Peierls correlations, and as the "in-plane" SDW ${ }^{x, y_{-}}$ correlations are seen to be incoherent we are left with

$$
\begin{aligned}
\left\langle\mathcal{O}_{S D W}^{z}(x) \mathcal{O}_{S D W}^{z}\left(x^{\prime}\right)\right\rangle \sim \cos \left(2 k_{F}\left(x-x^{\prime}\right)\right) & \\
& \rightarrow(-1)^{l} \times \text { constant }
\end{aligned}
$$

where in the last step we have reintroduced the discrete stripe coordinate $x=r a_{0}, x^{\prime}=(r+l) a_{0}$. Thus, given the hypothesis of a phase-antiphase spin background one obtains a long-ranged antiferromagnetic (Néel) phase for the stripe. The energy of this frustrated configuration grows linear with the length of the stripe and is hence unphysical, as anticipated. Note, however, that the actual "in-phase" Néel configuration for $n_{e}=1$ does imply a long-ranged Néel phase for the stripe!

- B phase: $0<U+\beta J_{K}^{2} / J_{H}<2 V$.

This phase is that of an insulator with a long-range ordered CDW: Both charge and spin excitations are gapped. The fields $\varphi_{c(s)}^{\prime}$ get ordered with ground state expectation values $\left\langle\varphi_{s}^{\prime}\right\rangle=0$ and $\left\langle\varphi_{c}^{\prime}\right\rangle=\sqrt{\pi / 8 K_{c}}$ respectively, and

$$
\left\langle\mathcal{O}_{C D W}(x) \mathcal{O}_{C D W}\left(x^{\prime}\right)\right\rangle \sim(-1)^{l} \times \text { constant },
$$

with $l$ defined after Eq. (95). For the case of an isolated stripe $\left(J_{K}=0\right)$, results from weak-coupling perturbative renormalization group studies ${ }^{55.65}$ show that there is a continuous phase transition along the line $U=2 \mathrm{~V}$ separating the $\mathrm{SDW}^{z}$ and $\mathrm{CDW}$ phases. The recent interest in the extended $U-V$ Hubbard model was triggered by Nakamura,$\underset{71}{,}$, who found numerical evidence that for small to intermediate values of $U$ and $V$, the $\mathrm{SDW}^{z}$ and 


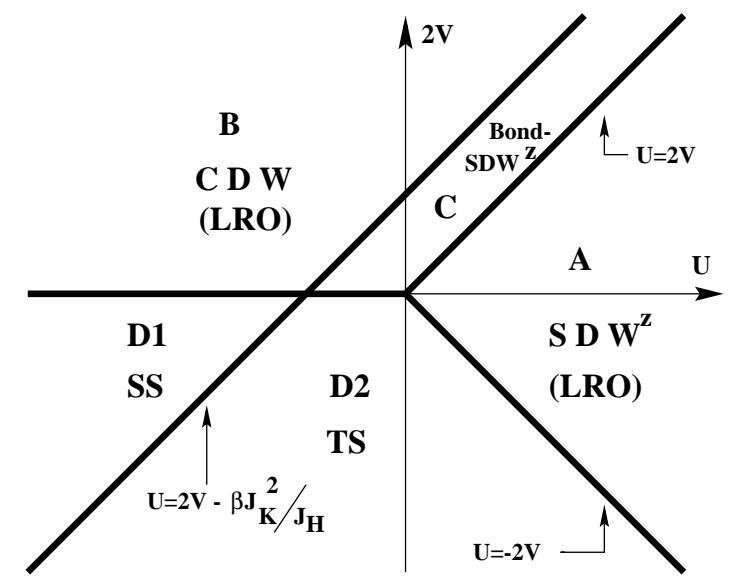

FIG. 4: The ground state phase diagram of the stripe electron system at half-filling. Solid lines separate different phases: $\mathrm{SDW}^{z}$ - long-range ordered spin density wave phase; CDW - long-range ordered charge density wave phase; SS - BCS (superconducting) singlet pairing phase; $\mathrm{TS}^{z}-$ triplet pairing phase (coexisting with a composite SS); $\mathrm{bSDW}^{z}$ - long-range ordered bond-located spin density wave. As explained in the text, the $A$-domain can only be realized with an "in-phase" Néel configuration across the stripe. The other stripe phases are assumed to coexist with antiphase Néel configurations of the localized spins.

CDW phases are mediated by a bond-located chargedensity-wave (bCDW) phase: The $\mathrm{SDW}^{z}$-CDW transition splits into two separate transitions: $(i)$ a KosterlitzThouless spin gap transition from $\mathrm{SDW}^{z}$ to bCDW and (ii) a continuous transition from bCDW to CDW. An analogous sequence of phase transitions in the vicinity of the $U=2 V$ line is an intrinsic feature of extended $U-V$ Hubbard models with bond-charge coupling 66 . A similar effect is here caused by the Kondo coupling to the antiferromagnetic environment: At $J_{K} \neq 0$, along the line $U=2 \mathrm{~V}$ only the charge gap closes. Therefore this line correspond to a metallic state with dominating antiferromagnetic $\mathrm{SDW}^{z}$ and $\mathrm{bSDW}^{z}$ correlations (since $K_{c}<1$ ), showing identical power-law decays at large distances:

$$
\begin{aligned}
\left\langle\mathcal{O}_{S D W}^{z}(x) \mathcal{O}_{S D W}^{z}\left(x^{\prime}\right)\right\rangle & =\left\langle\mathcal{O}_{b S D W}^{z}(x) \mathcal{O}_{b S D W}^{z}\left(x^{\prime}\right)\right\rangle \\
& \sim(-1)^{l} \times\left|x-x^{\prime}\right|^{-K_{c}} .
\end{aligned}
$$

- C phase: $\quad-\beta J_{K}^{2} / J_{H}<U-2 V<0$ and $V>0$.

Here again a charge gap opens. However, since in this sector $U-2 V<0$ the bosonic charge field is now condensed with ground state expectation value $\left\langle\varphi_{c}^{\prime}\right\rangle=\sqrt{\pi / 8 K_{c}}$. This immediately leads to suppression of the site-located $\mathrm{SDW}^{z}$ correlations, and instead the bond-located $\mathrm{bSDW}^{z}$ exhibits long-range order:

$$
\left\langle\mathcal{O}_{b S D W}^{z}(x) \mathcal{O}_{b S D W}^{z}\left(x^{\prime}\right)\right\rangle \sim(-1)^{l} \times \text { constant } .
$$

The line $V=0, U<0$ is the crossover line from the insulating phases into the superconducting phases. On this line the charge sector is in the weak-coupling gapless (metallic) phase with $K_{c}^{*}=1$. However, the spin sector is massive along this line except a the point $U=-\beta J_{K}^{2} / J_{H}$, which marks the transition from a metallic phase at $-\beta J_{K}^{2} / J_{H}<U<0$ where the $\mathrm{SDW}^{z}, \mathrm{bSDW}^{z}$ and $\mathrm{TS}^{z}$ fluctuations show identical algebraic decay at large distances

$$
\begin{array}{r}
\left\langle\mathcal{O}_{S D W}^{z}(x) \mathcal{O}_{S D W}^{z}\left(x^{\prime}\right)\right\rangle \sim\left\langle\mathcal{O}_{b S D W}^{z}(x) \mathcal{O}_{b S D W}^{z}\left(x^{\prime}\right)\right\rangle \\
\sim\left\langle\mathcal{O}_{T S}^{z}(x) \mathcal{O}_{T S}^{z}\left(x^{\prime}\right)\right\rangle \sim\left|x-x^{\prime}\right|^{-1},
\end{array}
$$

to a different metallic phase at $U<-\beta J_{K}^{2} / J_{H}$, where the SDW, bSDW and $\mathrm{TS}^{z}$ fluctuations are suppressed, while the conventional CDW, SS and Peierls correlations show identical large distance behavior:

$$
\begin{aligned}
\left\langle\mathcal{O}_{C D W}(x) \mathcal{O}_{C D W}\left(x^{\prime}\right)\right\rangle \sim\left\langle\mathcal{O}_{S S}(x) \mathcal{O}_{S S}\left(x^{\prime}\right)\right\rangle \\
\sim\left\langle\mathcal{O}_{\text {dimer }}(x) \mathcal{O}_{\text {dimer }}\left(x^{\prime}\right)\right\rangle \sim\left|x-x^{\prime}\right|^{-1} .
\end{aligned}
$$

This large degeneracy of metallic phases along the line $V=0$ is due to the $\mathrm{SU}(2)$-charge ("pseudo-spin") symmetry of the half-filled Hubbard model ${ }^{61}$. This degeneracy is immediately lifted by an attractive nearestneighbor coupling $(V<0)$, in support of superconducting instabilities. One finds two phases with enhanced pairing correlations:

- D1 phase: $U<2 V-\beta J_{K}^{2} / J_{H}$ and $V<0$.

Here the dominating instability is towards conventional BCS singlet pairing, with correlations

$$
\left\langle\mathcal{O}_{S S}(x) \mathcal{O}_{S S}\left(x^{\prime}\right)\right\rangle \sim\left|x-x^{\prime}\right|^{-1 / K_{c}} .
$$

- D2 phase: $2 V-\beta J_{K}^{2} / J_{H}<U<-2 V, \quad V<0$.

In this region triplet pairing shows a power-law decay at large distances

$$
\left\langle\mathcal{O}_{T S}^{z}(x) \mathcal{O}_{T S}^{z}\left(x^{\prime}\right)\right\rangle \sim\left|x-x^{\prime}\right|^{-1 / K_{c}},
$$

and is the dominating instability in the ground state. It follows that the composite singlet pairing operator $\mathcal{O}_{c-S S}^{\left(k=\pi / a_{0}\right)}$, defined in Eq. (93), also builds up largedistance correlations:

$$
\begin{aligned}
& \left\langle\mathcal{O}_{c-S S}^{\left(k=\pi / a_{0}\right)}(x) \mathcal{O}_{c-S S}^{\left(k=\pi / a_{0}\right)}\left(x^{\prime}\right)\right\rangle \\
\sim & (-1)^{\ell}\left\langle\mathcal{O}_{T S}^{z}(x) \mathcal{O}_{T S}^{z}\left(x^{\prime}\right)\right\rangle\left\langle n^{z}(x) n^{z}\left(x^{\prime}\right)\right\rangle \\
\sim & (-1)^{\ell} \times\left|x-x^{\prime}\right|^{-1 / K_{c}} .
\end{aligned}
$$

We have here used Eqs. (93) and (102), together with the property that the Néel order parameter, with $\left\langle\boldsymbol{n}(x) \boldsymbol{n}\left(x^{\prime}\right)\right\rangle=\left\langle n^{z}(x) n^{z}\left(x^{\prime}\right)\right\rangle=$ constant, defines the "out-of-plane" direction $\hat{z}$ along which the triplet pairing correlations are enhanced. Similarly, for a diagonal stripe one would have, using Eqs. (94) and (102):

$$
\left\langle\mathcal{O}_{c-S S}^{(k=0)}(x) \mathcal{O}_{c-S S}^{(k=0)}\left(x^{\prime}\right)\right\rangle \sim\left|x-x^{\prime}\right|^{-1 / K_{c}} .
$$


However, as shown by Coleman et al $\stackrel{70}{n}, k=0$ oddfrequency pairing is likely to be intrinsically unstable, and hence is not expected to compete with the conventional triplet pairing mode.

\section{Away from half-filling: $n_{e} \neq 1$}

We now turn to the physically more relevant case of a stripe with $n_{e} \neq 1$, assuming that $n_{e}$ is within the range where the stripe forms an antiphase domain wall between the Néel configurations $\left(n_{e} \leq 0.6\right.$ in the strong-coupling limit $J_{K} \sim J_{H}$, according to Refs. 56 and 57).

Away from half-filling the charge sector is always in the (weak coupling) Luttinger liquid metallic regime, controlled by the bare value of the Luttinger liquid parameter $K_{c}$. The phase diagram now splits into four sectors: A, B, D1 and D2 (see FIG. 5), separated by the line $U-2 V+\beta J_{K}^{2} / J_{H}=0$ (where the spin gap closes) and by the crossover line $U+6 V=0\left(K_{c}=1\right)$ separating the metallic phases with dominating density-density correlations at $K_{c}<1$ from those with dominating superconducting correlations at $K_{c}>1$.

When $U-2 V+\beta J_{K}^{2} / J_{H}>0$ the spin sector flows to strong coupling with the excitations condensing at $\left\langle\varphi_{s}^{\prime}\right\rangle=\sqrt{\pi / 8 K_{s}}$. This kills off the CDW as well as the "in-plane" SDW ${ }^{x, y}$-correlations. The $\mathrm{SDW}^{z}$ and $\mathrm{TS}^{z}$ correlations, on the other hand, survive the spin field ordering, and one finds

$$
\begin{aligned}
\left\langle\mathcal{O}_{S D W}^{z}(x) \mathcal{O}_{S D W}^{z}\left(x^{\prime}\right)\right\rangle & \sim\left|x-x^{\prime}\right|^{-K_{c}} \\
\left\langle\mathcal{O}_{T S}^{z}(x) \mathcal{O}_{T S}^{z}\left(x^{\prime}\right)\right\rangle & \sim\left|x-x^{\prime}\right|^{-1 / K_{c}} .
\end{aligned}
$$

Therefore, in the

- A phase: $\quad U-2 V+\beta J_{K}^{2} / J_{H}>0 ; \quad K_{c}<1$,

the $\mathrm{SDW}^{z}$ correlation is the dominating instability in the system (with $\mathrm{TS}^{z}$ subleading), while in the

$$
\text { - D2 phase : } U-2 V+\beta J_{K}^{2} / J_{H}>0 ; \quad K_{c}>1 \text {, }
$$

the triplet pairing $\left(\mathrm{TS}^{z}\right)$ fluctuations dominate (with $\mathrm{SDW}^{z}$ being subleading).

When $U-2 V+\beta J_{K}^{2} / J_{H}<0$ the spin sector flows to the other strong coupling regime, with the spin excitations condensing at $\left\langle\varphi_{s}^{\prime}\right\rangle=0$, the charge sector remaining in the weak-coupling metallic region. The correlations that exhibit algebraic decay are those of CDW and $\mathrm{SS}^{z}$ excitations, and one finds

$$
\begin{aligned}
\left\langle\mathcal{O}_{C D W}(x) \mathcal{O}_{C D W}\left(x^{\prime}\right)\right\rangle & \sim\left|x-x^{\prime}\right|^{-K_{c}}, \\
\left\langle\mathcal{O}_{S S}^{z}(x) \mathcal{O}_{S S}^{z}\left(x^{\prime}\right)\right\rangle & \sim\left|x-x^{\prime}\right|^{-1 / K_{c}} .
\end{aligned}
$$

It follows that in the

- B phase: $\quad U-2 V+\beta J_{K}^{2} / J_{H}<0 ; \quad K_{c}<1$, the CDW correlation is the dominating instability in the system (with SS subleading), while in the

- D1 phase : $\quad U-2 V+\beta J_{K}^{2} / J_{H}<0 ; \quad K_{c}>1$,

the conventional singlet-pairing BCS fluctuations are the most dominant (with the CDW fluctuations being subleading).

In the $\mathrm{A}$ and $\mathrm{D} 1$ phases the composite $\mathrm{CDW}^{z}$ and $\mathrm{SS}$ order parameters also build up large- distance correlations. In exact analogy with the D2 phase at half-filling, one obtains for a collinear stripe:

$$
\left\langle\mathcal{O}_{c-C D W}^{\left(k=2 k_{F}^{*}\right)}(x) \mathcal{O}_{c-C D W S}^{\left(k=2 k_{F}^{*}\right)}\left(x^{\prime}\right)\right\rangle \sim(-1)^{\ell} \times\left|x-x^{\prime}\right|^{-K_{c}},
$$

and

$$
\left\langle\mathcal{O}_{c-S S}^{\left(k=\pi / a_{0}\right)}(x) \mathcal{O}_{c-S S}^{\left(k=\pi / a_{0}\right)}\left(x^{\prime}\right)\right\rangle \sim(-1)^{\ell} \times\left|x-x^{\prime}\right|^{-1 / K_{c}} .
$$

For a diagonal stripe a composite CDW also develops, with correlations

$$
\left\langle\mathcal{O}_{c-C D W}^{\left(k=2 k_{F}\right)}(x) \mathcal{O}_{c-C D W S}^{\left(k=2 k_{F}\right)}\left(x^{\prime}\right)\right\rangle \sim\left|x-x^{\prime}\right|^{-K_{c}} .
$$

The fate of the zero-momentum composite singlet is more uncertain, however, considering its intrinsic instability ${ }^{70}$.

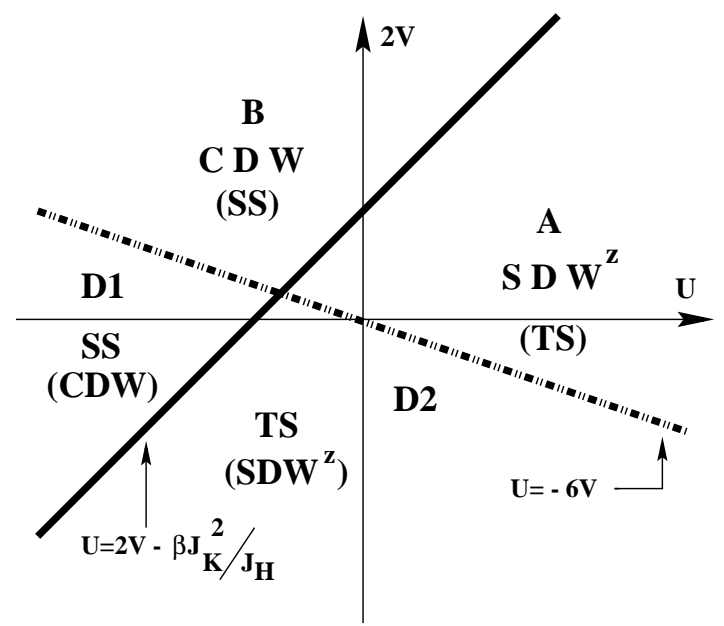

FIG. 5: The ground state phase diagram of the stripe electron system at $n_{e} \neq 1$. The solid line corresponds to a narrow metallic phase with gapless spin excitation spectrum, and separates two different spin gapped phases: the $\mathrm{SDW}^{z}$ and/or $\mathrm{TS}^{z}$ phase and the CDW and/or SS phase, respectively. The dashed line marks the crossover from a metallic phase with dominating density-density correlations into a phase with dominating pairing correlations. All instabilities shown in the phase diagram exhibit a power law decay of correlations. Subleading instabilities with correlations which decay faster then the dominating ones are indicated in brackets. As discussed in the text, the enhanced $\mathrm{SDW}^{z}\left(\mathrm{TS}^{z}\right)$ correlations coexist with composite CDW (SS) instabilities.

We summarize our findings in FIG. 5. We have to stress again the weak-coupling nature of the phase diagram. Higher-order corrections will modify the shape 
of the phase boundaries. However, more important are strong coupling effects. In the case of strong values of the Kondo lattice interaction, one may expect additional phase transitions due to the finite band width of the effective stripe model. Such effects cannot be traced within the continuum limit (infinite band) approach used in this paper, and will require numerical studies.

\section{The A phase away from half-filling: A scenario for nonconventional superconductivity?}

Of the phases considered, the A phase away from half-filling is of particular interest as the conditions $U-2 V+\beta J_{K}^{2} / J_{H}>0 ; K_{c}<1 ; n_{e} \neq 1$ are expected to apply to a generic stripe in a cuprate material: Most experiments ${ }^{72}$ indicate that the stripes in the cuprates are intrinsically metallic, with no commensurability gap even when the stripe order is static and strong, as in the Nd-doped materials ${ }^{73}$. Although a precise specification of the coupling constants is beyond present-day technology, the Coulomb interaction among the stripe electrons is expected to dominate other couplings (electronphonon, dopant potentials, interlayer fields,...), implying the bound $U-2 V+\beta J_{K}^{2} / J_{H}>0$, with $K_{c}<1$. Unfortunately, the electron dynamics on time scales where stripe fluctuations can be neglected (for which our model may apply) is still to be searched out experimentally, and there are as yet no "hard data" against which we can confront our results.

The A phase is dominated by a conventional $\mathrm{SDW}^{z}$ instability together with a composite CDW, coexisting with two subleading superconducting instabilities: conventional triplet pairing and composite singlet pairing (breaking parity and time reversal). This is different from the well-known scenario of a "spin gap proximity effect" $\underline{\underline{9}}$ where pair-hopping between a stripe and a spin-gapped insulating environment "infects" the stripe with the gap, resulting in a conventional CDW instability, with a subleading singlet pairing channel. In the case where stripe fluctuations are sufficiently slow that they can be treated as "quasi-static", the CDW instability can be shown to be suppressed by destructive interference between neighboring meandering stripes, leaving the singlet superconducting instability as the leading one ${ }^{10}$. The singlet order parameter on each stripe is then assumed to become correlated across the sample via inter-stripe "Josephson" coupling, leading to superconducting long-range order below a critical temperature. In contrast, in our scenario singlet superconductivity (with the added property of breaking parity and time reversal symmetry) would require the suppression of the leading $\mathrm{SDW}^{z}$ and (composite) CDW instabilities, in addition to that of triplet pairing. As we shall see below, quasi-static fluctuations do not perform this trick. Rather, meandering stripes living on a collinear backbone tend to phase lock so that (conventional) triplet pairing comes out as the leading effective instability. In the case of a diagonal structure, the (composite) singlet pairing correlations (if at all present; cf. our discussion after Eq. (94), survive the slow stripe fluctuations, and coexist with the triplet pairing channel. Whether a complete theory - treating stripe fluctuations and the one-dimensional electron dynamics on equal footing - would change our picture in favor of singlet pairing remains an open question.

Leaving for future work the problem if and how longrange superconducting order may emerge from an Aphase type instability when stripe fluctuations are fully included in the analysis, there are still several issues that need to be addressed:

- Is the spin gap sufficiently large for the instabilities in the A phase to survive at finite temperatures?

- What happens when taking into account the fact that the antiferromagnetic environment as seen by a stripe is not that of two semi-infinite domains but is rather made up of two finite-width domains, separating the stripe from its neighbors?

- How do transverse stripe fluctuations influence the A-phase instabilities?

- What about possible long-range interactions among the stripe electrons?

Let us discuss these questions in turn.

The size of the spin gap. The A phase corresponds to a strong-coupling regime $g_{s}=-\left|g_{\perp}\right|$ which is reached after a crossover from weak coupling (where $\left.g_{s}=\left|g_{\perp}\right|\right)$; cf. Sec. III.A and FIG. 3. Because of the crossover, the spin gap opens slowly, and it is a priori not obvious that it will suffice to sustain the A phase in presence of thermal fluctuations. To find out, we use (63) to integrate the RG equations (62), and identify the length scale where $\left|g_{\perp}\right|$ becomes of order unity. This scale - where the perturbation is of the same order as the fixed point Hamiltonian and renders the theory noncritical - defines the correlation length $\xi_{s}$ of the electronic spin sector. Using $\left|g_{\perp}\left(\xi_{s}\right)\right| \sim \mathcal{O}(1) \gg\left|g_{0 s}\right|$ in the integrated scaling equation for $g_{\perp}$, we obtain $\xi_{s}=a_{0} \exp \left[\left(\pi / 2-\arctan \left(g_{0 s} / \delta g\right)\right) / 2 \pi \delta g\right]$, where $\delta g \equiv$ $\sqrt{g_{0 \perp}^{2}-g_{0 s}^{2}}$. The associated spin gap $\Delta_{s}=\hbar v_{s} \xi_{s}^{-1}$ is thus given by

$$
\Delta_{s}=\hbar \frac{v_{s}}{a_{0}} \exp \left[-\frac{\pi / 2-\arctan \left(g_{0 s} / \sqrt{g_{0 \perp}^{2}-g_{0 s}^{2}}\right)}{2 \pi \sqrt{g_{0 \perp}^{2}-g_{0 s}^{2}}}\right],
$$

with $g_{0 s}=a_{0}(U-2 V) / \pi v_{s}$ and $g_{0 \perp}=a_{0}(U-2 V+$ $\left.\beta J_{K}^{2} / J_{H}\right) / \pi v_{s}$, as given in Eq. (55). There is considerable latitude in specifying the parameters entering (112), but choosing $v_{s} \sim 10^{5} \mathrm{~m} / \mathrm{s}, a_{0} \sim 5 \AA, U-2 V \sim 10^{-1} \mathrm{eV}$, and $J_{K}^{2} / J_{H} \sim 10^{-4} \mathrm{eV}$ - all within reasonable bounds - we obtain from (112) a spin gap $\Delta_{s}$ corresponding to a temperature of about $500 \mathrm{~K}$. We conclude that the gap is robust, and is expected to sustain the A phase in the relevant temperature range (where stripe formation 
is possible $\left.{ }^{36}\right)$. It is interesting to note that an estimate of $500 \mathrm{~K}$ is about "right" if one were to identify the spin gap with the pseudogap observed in the underdoped metallic phase of the cuprates ${ }^{35}$. A considerable amount of local pairing would then be present well beyond the superconducting transition temperature (in this region determined by the onset of global phase coherence).

Finite-width antiferromagnetic domains. A stripe in a real material is not embedded in a $2 \mathrm{D}$ antiferromagnet, but is separated from its neighbors by finite-width domains, or spin ladders, with a finite antiferromagnetic correlation length $\xi_{A F M}$. In order for the A phase to survive in this more harsh environment the spin gap must develop on a length scale shorter than $\xi_{A F M}$ (where the stripe electrons can still sample local magnetic order). This implies the condition

$$
\xi_{s}<\xi_{A F M}
$$

with $\xi_{s}$ the spin correlation length on the stripe. The zero-temperature correlation length $\xi_{A F M}$ for $S=1 / 2$ spin ladders with an even number of legs $n_{\ell}$ has been calculated analytically ${ }^{74}$ :

$$
\xi_{A F M} \approx 0.5 a_{0} \mathrm{e}^{0.68 n_{\ell}}\left(1-0.73 n_{\ell}\right)
$$

where, as before, $a_{0}$ is the lattice spacing. Using that $\xi_{s}=\hbar v_{s} / \Delta_{s}$, our estimate from above, $\Delta_{s} / k_{B} \sim 500 \mathrm{~K}$, together with (113) and (114), imply that the A phase survives for even-leg ladders with $n_{\ell} \geq 4$. As suggested by the Monte-Carlo data in ${ }^{75}$, a four-leg ladder with $J_{H}$ of the order of $10^{-2} \mathrm{eV}$ may support the $\mathrm{A}$ phase up to a temperature of $T \sim 50 \mathrm{~K}$. By increasing $U-2 V$ and/or $J_{K}$, the spin gap grows, allowing for the A phase to persist at the lower bound $n_{\ell}=4$ for even higher temperatures.

The case of an odd-leg ladder requires further analysis. Now, with $S=1 / 2$, the Berry phase in 18 contributes a nonvanishing topological term to the effective action for the spin ladder, implying a diverging spin-correlation length $\xi \rightarrow \infty$ but with no antiferromagnetic order over large distances. However, in the weak coupling regime the topological term is effectively inactive ${ }^{74}$, and as a consequence there is no distinction between gapless and gapful ladders on length scales shorter than $\xi_{A F M}$. It follows that the condition (113) is the same for evenleg and odd-leg ladders. Although we cannot rigorously exclude that nonperturbative effects may carry over to the stripe electrons on length scales larger than $\xi_{A F M}$, it seems improbable considering the fact that the spin sector of the stripe develops a mass at a length scale which is shorter than and independent of $\xi_{A F M}$. As such the mass is already well-established at the scale where non-perturbative effects from the ladder may come into play.

Before concluding this discussion we wish to add two more notes: First, the analysis sketched here is strictly valid only for a collinear stripe structure. As we commented upon in Sec II.B, the unusual boundary condition implied by a diagonal stripe orientation influences the Berry phase in a nontrivial way. This may produce a non-negligible feed-back on the localized spins when considering a finite-width or spin-ladder environment, possibly changing the A phase in an unexpected way. Secondly, one should note that the composite order parameters for a collinear structure, defined in Eqs. (90) and (93), decay faster with a spin-ladder environment as compared to the case of two semi-infinite $2 \mathrm{D}$ domains. It follows that at distances shorter than $\xi_{A F M}$ - where our construction is still expected to be valid - the algebraic decay of the Néel-order correlations produce a faster decay of the composite correlations compared to the conventional ones. This is different from the case where a one-dimensional electron gas is coupled by a Kondo lattice coupling to two non-interacting antiferromagnetic Heisenberg spin-1/2 chains (i.e. with $n_{\ell}=1$ ). As shown in Ref. 30, the composite order parameters (c-SS and c-CDW) here induce the dominant instabilities. This reflects the fact that the model with an $n_{\ell}=1$ environment renormalizes to a fixed point different from ours, instead belonging to the universality class of chirally stabilized liquids 31 , with no opening of a spin gap. It is here important to note that if one were to increase our model parameters $U-2 V$ and/or $J_{K}$ so that (113) and (114) were simultaneously satisfied for $n_{\ell}=1$, our construction would break down. In particular, the assumption of a weak coupling RG scheme - as exploited in Sec. III.A - would be violated. In addition, the pronounced role of the Berry phase for $n_{\ell}=1$ is likely to invalidate the construction of the effective spin sector model in (31), as paths in (17) away from the extremum now enter the stage to influence the ground state also at short and intermediates length scales. The $n_{\ell}=1$ environment is therefore not expected to be covered by our approach, and must instead be studied by other methods, such as that advocated in Ref. 30 .

The quasi-static limit: the effect of slow stripe fluctuations. To study the effect of slow stripe fluctuations on the order parameter correlations of phase A we consider a $2 \mathrm{D}$ array of static stripes and take an equilibrium average over their meanderings. Adopting the notation in Ref. 10, we use a coordinate system in which the stripe array runs along the $x$-direction (collinear or diagonal on the lattice), with a transverse displacement of a stripe in the $y$-direction labeled by $y_{j}(x)$, with $j$ indexing the stripe. Introducing the arc length $L_{j}(x)$, measuring the distance along the $j^{\text {th }}$ stripe to position $x$

$$
L_{j}(x)=\int_{0}^{x} d x^{\prime} \sqrt{1+\left(\partial_{x^{\prime}} y_{j}\left(x^{\prime}\right)\right)^{2}}
$$

we infer from Eq. (78) the expression for the $\mathrm{SDW}^{z}$ order parameter on a meandering stripe:

$$
\mathcal{O}_{S D W}^{z}(j, x)=\tilde{\mathcal{O}}_{S D W}^{z}(j, x)+h . c .
$$


with

$$
\tilde{\mathcal{O}}_{S D W}^{z}(j, x) \sim e^{i\left(2 k_{F} L_{j}(x)-\sqrt{2 \pi K_{c}} \varphi_{c}^{\prime}(j, x)\right)} \sin \left[\sqrt{2 \pi K_{s}} \varphi_{s}^{\prime}(j, x)\right]
$$

The coupling of the $\mathrm{SDW}^{z}$ to that of a neighboring stripe is of the form ${ }^{76}$

$$
\begin{aligned}
H_{S D W} & \sim \int d x g\left[\Delta y_{j}(x)\right] \quad\left(\tilde{\mathcal{O}}_{S D W^{z}}^{\dagger}(j, x) \tilde{\mathcal{O}}_{S D W^{z}}(j+1, x)+h . c .\right) \\
& \sim \int d x g\left[\Delta y_{j}(x)\right] \sin \left[\sqrt{2 \pi K_{s}} \varphi_{s}^{\prime}(j, x)\right] \sin \left[\sqrt{2 \pi K_{s}} \varphi_{s}^{\prime}(j+1, x)\right] \cos \left[\sqrt{2 \pi K_{c}} \Delta \varphi_{c}^{\prime}(j, x)-2 k_{F} \Delta L_{j}(x)\right](11
\end{aligned}
$$

where $g\left(\Delta y_{j}(x)\right)$ is an $x$-dependent coupling constant. Neglecting possible overhangs of stripes we have here defined $\Delta y(j, x) \equiv y(j+1, x)-y(j, x)>0$ (and similarly for $\Delta \varphi_{c}^{\prime}$ and $\left.\Delta L_{j}\right)$. By integrating out the stripe fluctuations $y_{j}(x)$ in powers of $g$ one obtains an effective Hamiltonian of an equivalent rigid system, with a renormalized coupling

$$
\tilde{g} \sim\left\langle g\left[\Delta y_{j}\right]\right\rangle \exp \left(-2 k_{F}^{2}\left\langle\left(\Delta L_{j}\right)^{2}\right\rangle\right)+\mathcal{O}\left(g^{2}\right),
$$

where $\langle\ldots\rangle$ denotes an average over meandering stripes. As argued in Ref. 10, since the signs of $\Delta L_{j}(x)$ are randomly distributed along the distance $x$, one expects $\Delta L_{j}(x)$ to grow as a random walk: $\left\langle\left(\Delta L_{j}(x)\right)^{2}\right\rangle \sim|x|$. It follows from (118) that the interstripe coupling between $\mathrm{SDW}^{z}$ 's can be ignored in the thermodynamic limit.

The conclusion that transverse stripe fluctuations cause destructive interference between $\mathrm{SDW}^{z}$ 's on neighboring stripes clearly applies to any $k \neq 0$ order parameter: The exponential suppression in (118) can be avoided only if the momentum transfer multiplying $\left\langle\left(\Delta L_{j}(x)\right)^{2}\right\rangle$ is identical to zero. Thus, the dephasing effect operates also for the A-phase composite CDW's, Eqs. (90) and (91) (with $k=2 k_{F}+\pi / a_{0}\left[k=2 k_{F}\right]$ for a collinear [diagonal] stripe backbone), as well as for the staggered $k=$ $\pi / a_{0}$ composite singlet pairing, Eq. (93), for a collinear structure ${ }^{77}$. In contrast, the conventional $(k=0)$ triplet pairing channel (88) survives the stripe fluctuations.

We caution the reader that the argument, adopted from Ref. 10, is valid only in the quasi-static limit where stripe fluctuations are sufficiently slow to be treated via an annealed average over (static) meandering stripes. Moreover, the argument is strictly valid only in the thermodynamic limit. The complete problem, where the quantum dynamics of mesoscopic stripes is treated on equal footing with the intrinsic Luttinger liquid instabilities remains unsolved.

For complementary views of the physics of meandering and fluctuating stripes we refer the reader to Refs. 78-82.

What about long-range electron-electron interactions? This question becomes critical when realizing that the Coulomb interaction on an isolated stripe is poorly screened, given its insulating environment. Neighboring stripes may provide metallic screening over a finite range, but our "assumption" that this range is of the order of a lattice spacing - implicitly built into the extended $U-V$ Hubbard model in (2) - may not be realistic. Still, having included a nearest-neighbor repulsion $V$ in the model, we do obtain some information about the effect of the poor screening: As can be gathered from Eqs. (55), (105) and (106), the presence of $V>0$ enhances the $\mathrm{SDW}^{z}$ instability, whereas the $\mathrm{TP}^{z}$ gets weaker. In the case of an arbitrary finite screening length $\kappa_{s}$, Schulz ${ }^{83}$ found that the large-distance correlations are governed by a modified charge parameter $K_{c} \sim 1 / \sqrt{\ln \kappa_{s}}$. In the A phase, this again gets translated into stronger (weaker) $\mathrm{SDW}^{z}\left(\mathrm{TP}^{z}\right)$ correlations as compared to the case with only local on-site interactions $\sim U$ (cf. again Eqs. (105) and (106) $)^{84}$. For small $J_{K}^{2} / J_{H}$ (as assumed here) one expects this result to apply also in the spin-gapped A phase. We conclude that as long as there is a finite screening length $\kappa_{s}$ present, our results - using the $U-V$ Hubbard model - should remain at least qualitatively valid for length scales $>\kappa_{s}$ : The A phase supports an $\mathrm{SDW}^{z}$ instability with a subleading $\mathrm{TS}^{z}$ (which, however, gets weaker as $\kappa_{s}$ grows larger). Taking into account the finite lengths of the stripes would introduce yet another scale into the problem (cf. the discussion above), requiring a more sophisticated analysis.

In this context we wish to remind the reader that we obtained the induced spin-interaction in (31) by a saddlepoint approximation, with $\boldsymbol{n} \times \partial_{\tau} \boldsymbol{n}$ in (27) locked to the small but fast oscillations of the $\boldsymbol{\ell}$-field. Fluctuations away from the extremum are expected to produce an effective retarded interaction in the spin sector, similar to what happens in the charge sector of a Luttinger liquid when integrating out electron-phonon interactions 85 . Whereas the local fluctuations of the Néel field could possibly be treated in analogy with the phonon problem ${ }^{85}$, an analysis of the dispersive antiferromagnetic excitations (which may produce a long-range tail of the retarded interaction) requires a novel theoretical approach. We have to leave this problem as a challenge for the future. 


\section{TWISTED ANTIFERROMAGNETIC DOMAINS}

So far we have have been concerned with the ideal situation of perfect phase-antiphase Néel configurations surrounding the stripe, giving rise to the effective $\mathrm{U}(1)$ symmetric model in (31). In this section we generalize the discussion to the case where fluctuations twist the Néel configurations with respect to each other, breaking the $\mathrm{SU}(2)$ spin-rotational symmetry completely.

How does the Néel order parameter change across the antiphase boundary defined by the stripe? Let us take the boundary along $y=0$. The simplest situation is that

$$
\langle\boldsymbol{n}(x, y)\rangle=f(y) \hat{z}
$$

with $f(y)=-f(-y)$. This is the situation we have considered so far; that the rotational symmetry about the spin $z$ axis is preserved, and that the Néel order parameter simply decreases across as we approach the antiphase boundary and is reversed on the opposite side.

A second possibility is that the Néel order parameter rotates along (for instance) the spin $x$ axis as it approaches the antiphase boundary. In addition, we can let it rotate around the spin $\mathrm{z}$ axis along the boundary, resulting in the following form for $\langle\boldsymbol{n}(x, y)\rangle$ :

$$
\langle\boldsymbol{n}(x, y)\rangle=(\cos (\alpha) \sin (2 \pi q x), \cos (\alpha) \cos (2 \pi q x), \sin (\alpha)),
$$

where $\alpha=\alpha(y)$ is an odd function with $\alpha( \pm \infty)= \pm \pi / 2$. The parameter $q$ is the rotational pitch along the antiphase boundary The special case $q=0$ corresponds to a "collinear" spin texture, and if $q \neq 0$, we find a phase that is topological, in the sense that the Neel order parameter covers the spin space with topological density of $4 \pi q$ per unit length of the phase boundary.

These phases are all further subdivided by their symmetry under reflections through the antiphase boundary. If the lattice points are arranged so that the line $y=0$ contains lattice points, the actual configuration is a configuration under reflections through $y=0$ and the antiphase boundary is site centered. If $y= \pm 1 / 2 a_{0}$ contains the lattice points, the configuration is even under reflections through $y=0$ and the configuration is bond centered.

These various stripe scenarios have all been investigated as candidates for spin textures associated with striped antiphase boundaries. Although the investigations are not conclusive, it is fair to say that neither experimental nor theoretical investigations suggest that anything but $q=0$ stripe configurations should be considered candidates for a spin texture. In fact, simple calculations $^{86}$ suggest that the spin texture that appears to be energetically favored is in fact the site centered collinear stripes along (10) that are odd under reflections through the antiphase boundary. For the diagonal (11) stripes, the bond centered and site centered have energies that are almost identical. Furthermore, the spin domains of these stripes are all extremely narrow, as measured by the width of the functions $\alpha(y)$. This supports our use of an effective 1D model for a site-centered stripe.

To study the effect of a completely broken spinrotational symmetry we shall confine our attention to the case of a collinear stripe (along the $\hat{x}$-direction) away from half-filling (cf. sec II.A), and with repulsive interactions among the electrons $(U-2 V>0)$. As before, we denote the two insulating semi-infinite domains surrounding the stripe by $A$ and $B$ respectively. We may assume that the Néel directions $\boldsymbol{n}^{(A)}(x, y)$ and $\boldsymbol{n}^{(B)}(x, y)$ are parameterized as in Eq. (120), with $q=0$ and with $\alpha$ a slowly varying function of $y$, except across the stripe where $\alpha$ changes sign. To connect to the local mean-field picture used in Sec. II.A we shall simply think of two fixed Néel directions $\left\langle\boldsymbol{n}^{(A)}\right\rangle$ and $\left\langle\boldsymbol{n}^{(B)}\right\rangle$ which differ by an arbitrary angle $\gamma, 0<\gamma<\pi$, across the stripe (with $\gamma=\pi$ for perfect phase-antiphase Néel configurations).

The construction of the effective model proceeds along the same lines as in Sec II.A However, since $\boldsymbol{n}_{r}^{(A)} \neq$ $-\boldsymbol{n}_{r}^{(B)}$ when $\gamma \neq \pi$, we must now pay attention to possible contributions from the coupling $H_{\boldsymbol{n}}$ of the lattice spin density to the Néel order parameters, Eq. (21). By inspection it is easy to verify that the staggered spin density $(-1)^{r} \boldsymbol{\Lambda}_{r}$ entering Eq. (21) vanishes in the continuum limit away from half-filling, implying that for this case $H_{\boldsymbol{n}}$ does not come into play, even when $\gamma \neq \pi$. Taking $\left\langle\boldsymbol{n}_{r}^{(A)}\right\rangle \rightarrow\left\langle\boldsymbol{n}(x)^{(A)}\right\rangle \sim \hat{z}$, as in sec II.A, it follows from (27) that the effective electron-electron interaction on the stripe mediated by the Kondo exchange with the $A$-domain remains the same as before, as given in Eq. (28):

$$
H_{i n t}^{(A)}=-\frac{a_{0} J_{K}^{2}}{2 J_{H}} \int d x\left(: J_{L}^{z} J_{L}^{z}:+: J_{R}^{z} J_{R}^{z}:+J_{L}^{x} J_{R}^{x}+J_{L}^{y} J_{R}^{y}\right)
$$

Turning to the $B$-domain, we rotate the coordinate system so that its Néel direction $\left\langle\boldsymbol{n}^{(B)}(x)\right\rangle$ lies in the $y z$ plane:

$$
\left\langle\boldsymbol{n}^{(B)}\right\rangle=\sin \gamma \hat{y}+\cos \gamma \hat{z}, \quad 0 \leq \gamma \leq \pi
$$

with $\gamma$ the angle w.r.t. the $\hat{z}$-axis defining the Néel order direction of the $A$-domain. The piece of the electron spin density $\boldsymbol{J}$ that survives the projection onto the plane in which the uniform $\ell^{(B)}$ components live (cf. the discussion in sec II.A) is given by

$$
\begin{aligned}
\boldsymbol{J}_{\perp} & \equiv \boldsymbol{J}-\left(\boldsymbol{J} \cdot\left\langle\boldsymbol{n}^{(B)}\right\rangle\right)\left\langle\boldsymbol{n}^{(B)}\right\rangle \\
& =\sin ^{2} \gamma J^{y} J^{y}+\cos ^{2} \gamma J^{z} J^{z} \\
& +\cos \gamma \sin \gamma\left(J^{y} J^{z}+J^{z} J^{y}\right) .
\end{aligned}
$$

Decomposing $\boldsymbol{J} \rightarrow \boldsymbol{J}_{L}+\boldsymbol{J}_{R}$, and using the chiral identities

$$
\begin{aligned}
& J_{L / R}^{y} J_{L / R}^{z}+J_{L / R}^{z} J_{L / R}^{y}=0 \\
& J_{L / R}^{y} J_{L / R}^{y}=J_{L / R}^{z} J_{L / R}^{z}
\end{aligned}
$$


it follows from (27) and (124) that the effective electronelectron stripe interaction mediated by the Kondo ex- change with the spins in the $B$ domain is given by

$$
H_{\text {int }}^{(B)}(\gamma)=-\frac{a_{0} J_{K}^{2}}{2 J_{H}} \int d x\left\{: J_{L}^{z} J_{L}^{z}:+: J_{R}^{z} J_{R}^{z}:+J_{L}^{x} J_{R}^{x}+\cos ^{2} \gamma J_{L}^{y} J_{R}^{y}+\sin ^{2} \gamma J_{L}^{z} J_{R}^{z}-\cos \gamma \sin \gamma\left(J_{L}^{y} J_{R}^{z}+J_{L}^{z} J_{R}^{y}\right)\right\} .
$$

The induced interaction terms (28) and (125) are now to be added to the spin Hamiltonian $H_{s}$ in (12) (which describes the spin sector of the stripe electrons decoupled from the environment). Writing $H_{s}+H_{\text {int }}^{(A)}+H_{\text {int }}^{(B)}(\gamma) \equiv H_{s}(\gamma)$, we obtain:

$$
\begin{aligned}
H_{s}(\gamma)=2 \pi v_{s} \int d x & \left\{: J_{L}^{z} J_{L}^{z}:+: J_{R}^{z} J_{R}^{z}:-g_{0 s}(\gamma) J_{L}^{z} J_{R}^{z}-g_{0 \perp} J_{L}^{x} J_{R}^{x}-\left(g_{0 \perp}-\frac{a_{0} J_{K}^{2}}{4 \pi v_{s} J_{H}} \sin ^{2} \gamma\right) J_{L}^{y} J_{R}^{y}\right. \\
& \left.+\frac{a_{0} J_{K}^{2}}{4 \pi v_{s} J_{H}} \cos \gamma \sin \gamma\left(J_{L}^{y} J_{R}^{z}+J_{L}^{z} J_{R}^{y}\right)\right\},
\end{aligned}
$$

with

$$
g_{0 s}(\gamma)=\frac{a_{0}}{\pi v_{s}}\left(U-2 V+\sin ^{2} \gamma \frac{J_{K}^{2}}{4 J_{H}}\right)
$$

and with $g_{0 \perp}$ and $v_{s}$ given in Eq. (32). Introducing the rotated currents

$$
j_{R / L}^{x}=J_{R / L}^{x}, \quad j_{R / L}^{y}=\cos (\theta) J_{R / L}^{y}+\sin (\theta) J_{R / L}^{z}, \quad j_{R / L}^{z}=-\sin (\theta) J_{R / L}^{y}+\cos (\theta) J_{R / L}^{z},
$$

we can write $H_{s}(\gamma)$ on diagonal form w.r.t. spin components by choosing $\theta=-\gamma / 2$ :

$$
H_{s}(\gamma)=2 \pi v_{s} \int d x\left\{: j_{L}^{z} j_{L}^{z}:+: j_{R}^{z} j_{R}^{z}:-g_{0 \perp}\left(j_{L}^{x} j_{R}^{x}+j_{L}^{y} j_{R}^{y}\right)-g_{0 s}(\gamma) j_{L}^{z} j_{R}^{z}+g_{0 f}(\gamma) j_{L}^{y} j_{R}^{y}\right\}
$$

where

$$
\begin{aligned}
g_{0 \perp} & =\frac{a_{0}}{\pi v_{F}}\left(U-2 V+\frac{J_{K}^{2}}{2 J_{H}}\right), \\
g_{0 s}(\gamma) & =\frac{a_{0}}{\pi v_{F}}\left(U-2 V+\sin ^{2}(\gamma / 2) \frac{J_{K}^{2}}{2 J_{H}}\right), \\
g_{0 f}(\gamma) & =\frac{a_{0}}{\pi v_{F}} \sin ^{2}(\gamma / 2) \frac{J_{K}^{2}}{2 J_{H}}, \\
v_{s} & =v_{F}-\frac{a_{0}}{2 \pi}\left(U-2 V+\frac{J_{K}^{2}}{J_{H}}\right) .
\end{aligned}
$$

The four first terms in (129) are of the same form as the spin Hamiltonian (31) for the ideal phase-antiphase $(\gamma=\pi)$ problem, but with a $\gamma$-dependent coupling $g_{0 s}(\gamma)$.
Introducing auxiliary fermion fields $\tilde{R}_{\mu}(x)$ and $\tilde{L}_{\mu}(x)$, connected to the rotated currents $j_{L / R}^{i}(x)$ by

$j_{L}^{i}=\frac{1}{2}: \tilde{L}_{\mu}^{\dagger} \sigma_{\mu \nu}^{i} \tilde{L}_{\nu}:, \quad j_{R}^{i}=\frac{1}{2}: \tilde{R}_{\mu}^{\dagger} \sigma_{\mu \nu}^{i} \tilde{R}_{\nu}:, \quad i=x, y, z$,

an application of the bosonization dictionary in Sec. II.C (with $L_{\mu} \rightarrow \tilde{L}_{\mu}, R_{\mu} \rightarrow \tilde{R}_{\mu}, J_{R / L}^{z} \rightarrow j_{R / L}^{z}$ ) produces, as expected, a sine-Gordon model for the corresponding bosonic spin field $\tilde{\varphi}_{s}$ and its dual $\tilde{\vartheta}_{s}$. Using the same procedure to bosonize also the last term in (129) which is new - we finally obtain

$$
H_{s}(\gamma)=\frac{v_{s}}{2} \int d x\left\{\left(\partial_{x} \tilde{\varphi}_{s}^{\prime}\right)^{2}+\left(\partial_{x} \tilde{\vartheta}_{s}^{\prime}\right)^{2}+\frac{2 m_{s}^{(\tilde{\varphi})}}{a_{0}^{2}} \kappa \cos \left(\sqrt{8 \pi K_{s}} \tilde{\varphi}_{s}^{\prime}\right)+\frac{2 m_{s}^{(\tilde{\vartheta})}}{a_{0}^{2}} \kappa \cos \left(\sqrt{8 \pi K_{s}^{-1}} \tilde{\vartheta}_{s}^{\prime}\right)\right\}
$$


where - to leading order in the coupling constants - we have:

$$
\begin{aligned}
2\left(K_{s}-1\right) & =g_{0 s}(\gamma)=\frac{a_{0}}{\pi v_{F}}\left(U-2 V+\frac{J_{K}^{2}}{2 J_{H}} \sin ^{2}(\gamma / 2)\right) \\
2 \pi m_{s}^{(\tilde{\varphi})} & =g_{0 \perp}(\gamma)=\frac{a_{0}}{\pi v_{F}}\left(U-2 V+\frac{J_{K}^{2}}{2 J_{H}}\left(1-\frac{1}{2} \sin ^{2}(\gamma / 2)\right)\right) \\
2 \pi m_{s}^{(\tilde{\vartheta})} & =g_{0 f}(\gamma)=-\frac{a_{0}}{\pi v_{F}} \frac{J_{K}^{2}}{4 J_{H}} \sin ^{2}(\gamma / 2) \\
v_{s} & =v_{F}-\frac{a_{0}}{2 \pi}\left(U-2 V+\frac{J_{K}^{2}}{J_{H}}\right)
\end{aligned}
$$

Thus, the spin sector of the stripe electrons in the presence of twisted antiferromagnetic domains is described by a generalized sine-Gordon model containing a cosine of the dual spin field $\vartheta_{s}^{\prime}$. The presence of this term allows for spin-nonconserving processes, reflecting the complete breaking of the spin rotational symmetry (down to an Ising like $Z_{2} \times Z_{2} \times Z_{2}$ discrete symmetry, with one $Z_{2}$ factor for each spin component in the rotated basis (128)). This model was first studied by Giamarchi and Schulz ${ }^{87}$, who used it to account for spin-orbit and electronic dipole-dipole interactions in quasi-one-dimensional conductors. Its perturbative RG equations (valid in the limit of small parameters $g_{0 s}(\gamma), g_{0 \perp}(\gamma)$ and $g_{0 f}(\gamma)$ are most easily derived by exploiting the operator product expansion 61 for the rotated currents in Eq. (128), using (129), and one obtains:

$$
\begin{aligned}
& \frac{d g_{s}}{d \ell}=g_{f}^{2}-g_{\perp}^{2}, \\
& \frac{d g_{\perp}}{d \ell}=-g_{s} g_{\perp}, \\
& \frac{d g_{f}}{d \ell}=g_{s} g_{f} .
\end{aligned}
$$

The renormalized couplings $g_{i} \equiv g_{i}(\gamma, \ell),(i=s, \perp, f)$ are connected to the bare parameters $g_{0 i}(\gamma)$ in (134) by $g_{i}(\gamma, \ell=0) \equiv g_{0 i}(\gamma)$, with $\ell=\ln \left(a / a_{0}\right)$, where $a$ is a renormalized length. Focusing on the case with repulsive electron-electron interaction $\left(U-2 V+J_{K}^{2} / 2 J_{H}>0\right.$, with $K_{c}<1$ ), we read off from (134) that $g_{0 s}(\gamma)>0$. For this case the RG equations (134) support two strong-coupling massive phases:

$$
\begin{gathered}
(i) g_{0 s}(\gamma)<g_{0 \perp}(\gamma)-\left|g_{0 f}(\gamma)\right|: \\
g_{s} \rightarrow-\infty,\left|g_{\perp}\right| \rightarrow \infty, g_{f} \rightarrow 0
\end{gathered}
$$

and

$$
\begin{gathered}
(i i) g_{0 s}(\gamma)>g_{0 \perp}(\gamma)-\left|g_{0 f}(\gamma)\right|: \\
g_{s} \rightarrow \infty,\left|g_{f}\right| \rightarrow \infty, g_{\perp} \rightarrow 0
\end{gathered}
$$

By inspection, using (134), we can label the two regions by the range of the twist angle $\gamma$ :
(i) $0<\gamma<\pi / 2$
(ii) $\pi / 2<\gamma<\pi$.

In region (i) $\left|g_{s}\right|$ and $\left|g_{\perp}\right|$ increase upon renormalization, while $g_{f}$ scales to zero. The dual spin field is hence irrelevant and the picture emerging is qualitatively the same as in the A phase of Sec. III.C.2: the $\mathrm{SDW}^{z}$ correlation is the leading instability (critical exponent $K_{c}$ ), with $\mathrm{TS}^{z}$ being subleading (critical exponent $1 / K_{c}$ ). Note, however, that the ordering tendency is now along the $z$-direction of the rotated frame, defined by the transformation in (128). It follows that for a twist angle $0 \leq \gamma<\pi / 2$, the leading (subleading) instability is that of a spin density wave (triplet pairing) oriented along a line in the plane spanned by $\left\langle\boldsymbol{n}^{(A)}\right\rangle$ and $\left\langle\boldsymbol{n}^{(B)}\right\rangle$, and making an angle $\gamma / 2$ with $\left\langle\boldsymbol{n}^{(A)}\right\rangle$.

Turning to region (ii), the renormalized coupling constants $g_{s}$ and $\left|g_{f}\right|$ are seen to flow to infinity, while $g_{\perp}$ scales to zero. For our case, where $g_{0 f}(\gamma)>0$, it follows that $\tilde{\vartheta}_{s}$ gets ordered with expectation value $\left\langle\tilde{\vartheta}_{s}\right\rangle=0$, while the spin field $\tilde{\varphi}_{s}$ stays disordered. By running down the list of bosonized order parameters in Sec III.B.1, we pinpoint the leading (subleading) instability as that of the $\mathrm{SDW}^{y}\left(\mathrm{TS}^{y}\right)$ correlation, with critical exponent $K_{c}\left(1 / K_{c}\right)$. Note that also this result refers to the rotated frame defined by (128). Translating back to the original frame we infer that for a twist angle $\pi / 2<\gamma \leq \pi$, the leading (subleading) instability is again that of a spin density wave (triplet pairing) oriented along an axis contained in the $\left\langle\boldsymbol{n}^{(A)}\right\rangle-\left\langle\boldsymbol{n}^{(B)}\right\rangle$-plane, but now making an angle $\gamma / 2+\pi / 2$ with $\left\langle\boldsymbol{n}^{(A)}\right\rangle$.

Two comments are here appropriate. First note that the axis which defines the enhanced SDW and TS correlations makes a $\pi / 2$-jump as the twist angle $\gamma$ passes through $\pi / 2$. This may appear reminiscent of a spin-flop transition induced by a magnetic field, as seen in certain quasi-1D materials 87 . However, there is no physical effect associated with the jump other than a sudden change of the direction along which the SDW and TS correlations are enhanced. Secondly, when $\gamma=\pi$ we do recover the result for the A phase in Sec III.C.2 (ideal phaseantiphase configuration). Note that the presence of the dual cosine-term in the effective model (132) simply reflects the fact that we are now working in the rotated frame. By undoing the rotation in (128) we immediately recover the standard sine-Gordon model, as given in (52) for a perfect phase-antiphase configuration. 
To summarize; we have shown that the opening of a Néel twist angle $\gamma \neq \pi$ across the stripe does not remove the instabilities of the A phase found in Sec. III.C.2. The conventional leading (subleading) instability is still that of a spin density wave (triplet pairing), but now tilted w.r.t. to the two Néel- directions.

By inspection of the composite order parameters defined in (89) and (92) it is clear that their amplitudes will drop as $\gamma$ decreases from its largest value $\gamma=\pi$, defining a perfect phase-antiphase Néel configuration. It is here interesting to note that when $\gamma \neq \pi$ there is room for additional composite order parameters. Besides the trivial variations of (89) and (92) where $\boldsymbol{S}^{(A)}-\boldsymbol{S}^{(B)} \rightarrow$ $\boldsymbol{S}^{(A)}+\boldsymbol{S}^{(B)}$, we can construct two composite vector order parameters:

$$
\begin{aligned}
\mathcal{O}_{c-S D W} & \sim \mathcal{O}_{S D W} \times\left(\boldsymbol{S}^{(A)} \times \boldsymbol{S}^{(B)}\right) \\
& \rightarrow \mathcal{O}_{S D W} \times\left(\boldsymbol{n}^{(A)} \times \boldsymbol{n}^{(B)}\right)
\end{aligned}
$$

and

$$
\begin{aligned}
\mathcal{O}_{c-T S} & \sim \mathcal{O}_{T S} \times\left(\boldsymbol{S}^{(A)} \times \boldsymbol{S}^{(B)}\right) \\
& \rightarrow \mathcal{O}_{T S} \times\left(\boldsymbol{n}^{(A)} \times \boldsymbol{n}^{(B)}\right),
\end{aligned}
$$

where in the second lines we have dropped the small contributions from the $\boldsymbol{\ell}$-fields (which can be neglected in the correlation functions, cf. Sec. III.B). These composites have the same symmetries as the corresponding conventional order parameters, SDW and TS, respectively. In particular, $\mathcal{O}_{c-T S}$ is even under time and spin reversal, while odd under a parity transformation. It follows that for a sufficiently rigid spin texture with $\gamma$ away from 0 and $\pi$, triplet pairing comes in two guises: one conventional mode and one composite mode, both carrying zero momentum. These pairing modes have enhanced correlations along well-defined directions, orthogonal to each other. As $\gamma \rightarrow \pi$ (perfect antiphase boundary) or $\gamma \rightarrow 0$ ("in-phase" boundary), the composite triplet channel gets suppressed and one is left with the pairing instabilities characterizing the A phase in Sec. III. Elsewhere we shall explore the consequences of this intriguing possibility.

\section{SUMMARY}

In this article we have analyzed the problem of a onedimensional electron liquid (charge stripe) embedded in a two-dimensional antiferromagnetic insulator, and coupled to it via a weak spin exchange. Using a mean-field type construction, the spin exchange gets encoded as an effective anisotropic spin interaction among the electrons. This interaction is shown to be marginally relevant under certain conditions (in a renormalization group sense), producing enhanced pairing and density fluctuations in the electron liquid.

For realistic values of the model parameters - assuming a screened Coulomb interaction for the electrons, and a conduction band away from half-filling - the dominant instabilities are towards a conventional spin density and a composite charge density wave, coexisting with subleading conventional triplet and composite singlet pairing correlations. Taking into account the slow transverse fluctuations of a stripe, the triplet pairing instability is expected to turn into the dominant one. While the magnitudes of the conventional instabilities do not change with the relative orientation of the Néel directions in the two domains surrounding the stripe, the composite correlations have largest amplitudes when the stripe forms an antiphase domain wall in the antiferromagnet. With the possible exception of the composite singlet pairing mode, the instabilities are found to be insensitive to the spatial orientation of the stripe on the underlying lattice (collinear or diagonal).

Our study has been motivated by a wish to understand the role of spin exchange between stripes and their environment in the cuprate superconductors. As is wellknown, these materials are exceedingly complex systems. It is unlikely that a simplified model like ours - where several aspects of the problem have been simply ignored can produce accurate predictions about experimental results. We have discussed a posteriori some of the effects not included in our model: the role of slow stripe fluctuations and interstripe couplings, the finite width of the antiferromagnetic domains encountered in real materials, as well as the expected poor screening of the Coulomb interaction along a stripe. Other aspects of the problem are yet to be addressed; most importantly the possible appearance of a retarded nonlocal spin interaction coming from large-amplitude fluctuations of the Néel order parameters. Also, the finite-size and boundary effects implied by the mesoscopic scale at which the stripes live need to be carefully studied.

The virtue of our "stripped-down" model is that it allows us to carry out a well-controlled analytical study, and as such it could serve as a stepping stone for more detailed investigations. The model predicts that a stripeenvironment spin exchange under certain conditions may produce instabilities towards pairing of electrons. This result is obtained without the assumption of a pre-existing spin gap in the insulating environment. When including effects from the fluctuations of the stripes the symmetry of the dominant pairing instability appears to come out "wrong" - as judged against available experimental evidence from the cuprates. Does this imply that a stripe-environment spin exchange plays no role for superconductivity in these materials? Or, could the picture change when properly building more dynamical elements into the model? To answer these questions will require more work.

\section{Acknowledgments}

We thank S. Östlund for a contribution on spin textures in two-dimensional antiferromagnets, and for kindly 
allowing us to use it in Sec. IV. It is also a pleasure to thank I. Affleck, N. Andrei, M. Granath, C. Morais Smith, B. Normand, and J. Voit for helpful discussions. GIJ acknowledges support by the SCOPES grant
N 7GEPJ62379. The work of HJ was supported by the Swedish Research Council, grant 621-2002-4947.
1 J. M. Tranquada et al., Nature 375, 651 (1995); G. Aeppli et al., Science 178, 1432 (1997); K. Yamada et al., Phys. Rev. B 57, 6165 (1998); H. Mook et al., Nature 401, 145 (1999).

2 Z. X. Shen et al., Science 280, 259 (1998).

${ }^{3}$ F. Borsa et al., Phys. Rev. B 52, 7334 (1995).

4 T. Suzuki et al., Phys. Rev. B 57, R3229 (1998); A. W. Hunt et al., Phys. Rev. Lett. 82, 4300 (1999).

5 C. H. Chen, S.-W. Cheong, and A. S. Cooper, Phys. Rev. Lett. 71, 2461 (1993); J. M. Tranquada et al. Phys. Rev. B 52, 3581 (1995).

6 S. Mori, C. H. Chen, and S.-W. Cheong, Nature 392, 473 (1998).

7 J. Zaanen and O. Gunnarsson, Phys. Rev. B 40, 7391 (1989); H. J. Schulz, J. Physique 50, 2833 (1989); D. Poilblanc and T. M. Rice, Phys. Rev. B 39, 9749 (1989); K. Machida, Physica C 158, 192 (1989); M. Kato et al., J. Phys. Soc. Jpn. 59, 1047 (1990).

8 V. J. Emery and S. A. Kivelson, Physica C 209, 597 (1993); U. Löw et al., Phys. Rev. Lett. 72, 1918 (1994); L. Chayes et al., Physica A 115, 129 (1996).

${ }^{9}$ V. J. Emery, S. A. Kivelson and O. Zachar, Phys. Rev. B 56, 6120 (1997).

10 S. A. Kivelson, E. Fradkin and V. J. Emery, Nature 393, 550 (1998); V. J. Emery et al., Phys. Rev. Lett 85, 2160 (2000).

11 S. R. White and D. J. Scalapino, Phys. Rev. Lett. 80, 1272 (1998); Phys. Rev. B 60, R753 (1999).

12 For a review, see J. Voit, Rep. Prog. Phys. 58, 977 (1995).

13 A. H. Castro Neto, Phys. Rev. Lett. 78, 3931 (1997); A. H. Castro Neto and F. Guinea, Phys. Rev. Lett. 80, 4040 (1998).

14 S. Sachdev and R. Shankar, Phys. Rev. B 38, 826 (1988).

15 Yu. A. Krotov, D.-H. Lee and A. V. Balatsky, Phys. Rev. B 56, 8367 (1997).

16 M. Granath and H. Johannesson, Phys. Rev. Lett. 83, 199 (1999).

17 C. M. Varma and A. Zawadowski, Phys. Rev. B 32, 7399 (1985); L. Balents and M. P. A. Fisher, Phys. Rev. B 53, 12133 (1996).

18 E. Dagotto and T. M. Rice, Science 271, 618 (1996); T. M. Rice, S. Haas, M. Sigrist, and F.-C. Zhang, Phys. Rev. B 56, 14655 (1997); S. R. White and D. J. Scalapino, Phys. Rev. B 57, 3031 (1998).

19 A. H. Castro Neto, C. de C. Chamon and C. Nayak, Phys. Rev. Lett. 79, 4629 (1997).

20 V. J. Emery, S. A. Kivelson, and O. Zachar, Phys. Rev. B 59, 15641 (1999); E. W. Carlson, D. Orgad, S. A. Kivelson, and V. J. Emery, Phys. Rev. B 62, 3422 (2000).

21 O. Zachar, S. A. Kivelson and V. J. Emery, Phys. Rev. Lett. 77, 1342 (1996).

22 S. Fujimoto and N. Kawakami, J. Phys. Soc. Jpn. 63, 4322 (1994).

23 S. R. White and I. Affleck, Phys. Rev. B 54, 9862 (1996); A. E. Sikkema, I. Affleck and S. R. White, Phys. Rev. Lett.
79, 929 (1997).

${ }^{24}$ W. J. Zheng, Phys. Rev. Lett. 83, 3534 (1999); Phys. Rev. B 62, 4042 (2000).

25 O. Zachar and A. M. Tsvelik, Phys. Rev. B 64, 033103 (2001).

26 O. Zachar, Phys. Rev. B 63, 205104 (2001).

27 V. J. Emery and S. Kivelson, Phys. Rev. B 46, 10812 (1992).

28 P. Coleman, A. Georges, and A. M. Tsvelik, J. Phys.: Condens. Matter 9, 345 (1997).

29 A. Balatsky and E. Abrahams, Phys. Rev. B 45, 13125 (1992); A. V. Balatsky and J. Bonča, Phys. Rev. B 48, 7445 (1993).

30 P. Azaria and P. Lecheminant, Phys. Rev. B 62, 61 (2000); Nucl. Phys. B 575, 439 (2000).

31 N. Andrei, M. R. Douglas, and A. Jerez, Phys. Rev. B 58, 7619 (1998).

32 A. Kaminski et al., Nature 416, 610 (2002).

33 For a review, see M. V. Sadovskii, Usp. Fiz. Nauk. 171, 539 (2001).

34 Note, however, that an odd-frequency gap function itself cannot serve as an order parameter since its equal-time expectation value vanishes. Rather, some moment of the gap function with frequency could be an appropriate order parameter.

35 For a comprehensive review of stripes and hightemperature superconductivity, including the issue of dimensional crossovers, see E. W. Carlson, V. J. Emery, S. A. Kivelson, and D. Orgad, cond-mat/0206217

36 J. M. Tranquada et al., J. Phys. Chem. Solids 60,1019 (1999).

37 J. M. Tranquada et al., Phys. Rev. B, 55, 6113 (1997).

38 S. Wakimoto et al., Phys. Rev. B 60, R769 (1999); Phys. Rev. 61, 3699 (2000).

39 M. Matsuda et al., Phys. Rev. B 62, 9148 (2000).

40 M. G. Zacher, R. Eder, E. Arrigoni, and W. Hanke, Int. J. Mod. Phys. B, 14, 3783 (2000).

41 A. Ino et al., Phys. Rev. B 62, 4137 (2000).

42 For a review, see for example G. Sierra in Proc. of the 1996 El Escorial Summer School on Strongly Correlated Magnetic and Superconducting Systems, cond-mat/9610057

43 Note that the viability of pair hopping in addition requires that the charge gap of the insulating environment is sufficiently small so as not to obstruct the process.

44 M. Fabrizio and A. O. Gogolin, Phys. Rev. B 51, 17827 (1995); A. E. Mattsson, S. Eggert, and H. Johannesson, Phys. Rev. B 56, 15615 (1997).

45 V. J. Emery, S. A. Kivelson, and J. M. Tranquada, Proc. Natl. Acad. Sci. USA 96, 3814 (1999).

46 For a review, see M. Grioni and J. Voit, in Electron Spectroscopies Applied to Low-Dimensional Materials, edited by H.P. Hughes and H.I. Starnberg (Kluwer, Dordrecht, 2000), p. 201.

47 See, for example, O. M. Auslaender, et al., Phys. Rev. Lett. 84, 1764 (2000), and references therein. 
48 See, for example, M. Grayson et al., Phys. Rev. Lett 86, 2645 (2001), and references therein.

49 The $\pi$-shifted correlations across the stripe are mediated via the interaction with the stripe electrons which induce an effective interaction between the $A$ - and $B$-domains. See Refs. 56 and 57.

50 R. Micnas, J. Ranninger, and S. Robaszkiewicz, Rev. Mod. Phys. 62, 113, (1990).

${ }^{51}$ For an introduction to the 1D Kondo lattice problem, see A. M. Tsvelik, Field Theory in Condensed Matter Physics (Cambridge University Press, Cambridge, 1996).

52 C. Buhler, S. Yunoki, and A. Moreo, Phys. Rev. Lett. 84, 2690 (2000).

${ }^{53}$ For a review, see e.g. E. Fradkin, Field Theories of Condensed Matter Systems (Addison-Wesley, Redwood City (1991)).

54 For a detailed exposition, see T. Einarsson and H. Johannesson, Phys. Rev. B 43, 5867 (1991).

${ }^{55} \mathrm{~V}$. J. Emery in Highly Conducting One-Dimensional Solids, edited by J.T. Devreese, R.P. Evrard, and V.E. Van Doren (Plenum, New York, 1979).

56 O. Zachar, Phys. Rev. B 65, 174411 (2002).

57 W. V. Liu and E. Fradkin, Phys. Rev. Lett. 86, 1865 (2001).

58 The property that only the $\ell$-field couples manifestly to the stripe also for a half-filled band uniquely depends on the stripe being "topological" (i.e. separating two phaseantiphase Néel domains) and does not apply to the standard Heisenberg-Kondo lattice mode ${ }^{151}$ or its extension in Ref. 16.

59 F. D. M. Haldane, Phys. Rev. Lett. 61, 1029 (1988).

60 This is different from more conventional approaches, where a magnetically ordered background is simulated by a static field, coupling directly to the spins of the charge carriers. See e.g. E. Langmann et al., Physica C 296, 119 (1998).

61 A. O. Gogolin, A. A. Nersesyan and A. M. Tsvelik, Bosonization and Strongly Correlated Systems (Cambridge University Press, Cambridge, 1998).

62 D. Sénéchal, cond-mat/9908262

${ }^{63}$ P. Wiegmann, J. Phys. C 11, 1583 (1978).

64 T. Giamarchi and H. J. Schulz, Phys. Rev. B 39, 4620 (1989).

65 J. Voit, Phys. Rev. B 45, 4027 (1992).

66 G. I. Japaridze, Phys. Lett. A 201, 239 (1995); G. I. Japaridze and A. P. Kampf, Phys. Rev. B 59, 12822 (1999).

67 G. I. Japaridze and E. Müller-Hartmann, Phys. Rev. B 61,
9019 (2000).

68 C. N. Yang, Phys. Rev. Lett. 63, 2144 (1989).

69 M. Yamanaka, M. Oshikawa, and I. Affleck, Phys. Rev. Lett 79, 1110 (1997).

70 P. Coleman, E. Miranda, and A. Tsvelik, Phys. Rev. B 49, 8955 (1994).

71 M. Nakamura, J. Phys. Soc. Japan 68, 3123 (1999); M. Nakamura, Phys. Rev. B 61, 16377 (2000); K. Itoh, M. Nakamura, and N. Muramoto, cond-mat/0102497

72 A. N. Lavrov et al., Phys. Rev. Lett. 87, 017007 (2001).

73 Y.Ando et al., Phys. Rev. Lett. 88, 137005 (2002).

74 S. Chakravarty, Phys. Rev. Lett. 77, 4446 (1996).

75 O. F. Syljuåsen, S. Chakravarty and M. Greven, Phys. Rev. Lett. 78, 4115 (1997).

76 Note that by coupling $\tilde{\mathcal{O}}_{S D W}^{\dagger}(j, x)$ and $\tilde{\mathcal{O}}_{S D W}^{z}(j+1, x)$ we eliminate Umklapp processes that would have resulted from the coupling of the "full" $\mathrm{SDW}^{z}$ order parameters $\mathcal{O}_{S D W^{z}}^{\dagger}(j, x)$ and $\mathcal{O}_{S D W^{z}}(j+1, x)$; cf. Eq. (78).

77 The staggering term $\pi / a_{0}$ in the momentum transfer $k$ for a composite order parameter is defined $\bmod \left(\pi y_{j}(x) / a_{0}^{2}\right)$. This reflects the local phase flip suffered by the Néel order directions adjacent to a stripe when it meanders out an odd number of lattice sites in the transverse direction. This does not change the dephasing argument for a staggered composite order parameter.

78 C. Nayak and F. Wilczek, Phys. Rev. Lett. 78, 2465 (1997).

79 C. M. Smith et al., Phys. Rev. B 58, 453 (1998).

80 H. Eskes et al., Phys. Rev. B 58, 6963 (1998); J. Zaanen, O. Y. Osman and W. van Saarloos, Phys. Rev. B 58, R11868 (1998).

81 O. Tchernyshyov and L. P. Pryadko, Phys. Rev. B 61, 12503 (2000).

82 A. H. Castro Neto, Phys. Rev. B 64, 104509 (2001).

83 H. J. Schulz, Phys. Rev. Lett. 71, 1864 (1993).

${ }^{84}$ For an unscreened Coulomb potential Schulz ${ }^{83}$ showed that the pairing correlations in a Luttinger liquid decay like $\exp \left[-\operatorname{const}(\ln x)^{-3 / 2}\right]$, i.e. faster than any power law. The leading instability is now that of a $k=4 k_{F}$ charge density wave, causing an instability towards a Wigner crystal.

85 J. Voit and H. J. Schulz, Phys. Rev. B 34, 7429 (1986).

86 S. Östlund and M. Andersson, Phys. Rev. B 65, 094408 (2002); M. Andersson and S. Östlund, cond-mat/0203485

87 T. Giamarchi and H. J. Schulz, J. Phys. France 49, 819 (1988). 